\title{
Heuristic Optimization of Group Structure Using Physics-Based Fitness Approximation
}

\author{
C. Yï ${ }^{*}$, G. Sjoden ${ }^{1}$, C. Edgar \\ *Corresponding author \\ ${ }^{1}$ Now at Air Force Technical Applications Center \\ Georgia Institute of Technology, Atlanta, GA, 30313 \\ E-Mails: yice@gatech.edu (C. Yi), glenn@gesjodencorp.com (G. Sjoden), cedgar7@gatech.edu (C.Edgar) \\ Address: 770 State St, Boggs Building Rm 3-09, Atlanta, GA 30332-0745, United States
}

\begin{abstract}
In Multi-Group (MG) deterministic radiation transport simulation applications, Fine-Group (FG) cross section libraries are often collapsed, or down-sampled into BroadGroup (BG) libraries to reduce computational cost. Previously, we developed a heuristic approach to determine the optimal BG group structure using the Particle Swarm Optimization (PSO) algorithm. A key step in PSO is to evaluate the fitness function, which measures the optimality, or fitness of a given group structure. In this paper, we demonstrated that a Simulation-Driven Fitness Function (SDFF) can be readily formulated by performing transport simulations on a BG structure, and then comparing the results, such as flux distribution, detector response, and/or k-effective, with the reference FG simulation solution. While the PSO approach is very effective in searching for global optimum in a very large solution space, the SDFF evaluation process can be very time-consuming since it requires a large number of $\mathrm{BG}$ transport simulations, even though a single $\mathrm{BG}$ simulation usually costs much less time than the reference FG calculation. To overcome this problem, a novel Physics-Based Fitness Approximation (PBFA) approach is developed based on the contributon transport theory. Compared to SDFF, PBFA does not require BG transport simulations. Therefore, it significantly reduces the computational cost for evaluation of the fitness function. We performed a thorough numerical test on a neutron detector model, the Dual Range Coincidence Counter, to examine the efficacy of PBFA. We found that a strong positive correlation exists between SDFF and PBFA. Thanks to this new finding, we can replace PBFA with SDFF to accelerate the PSO algorithm for the group structure optimization application.
\end{abstract}

Highlights: A Physics-Based Fitness Approximation method is developed based on the contributon transport theory for heuristic optimization of broad group structure. The new approach is compared with the Simulation-Driven Fitness Function on the Dual Range Coincidence Counter model.

Keywords: Group Structure Optimization, Particle Swarm Optimization, Contributon Transport, Physics-Based Fitness Approximation 


\section{Introduction}

Group constants are used as coefficients in the energy- domain discretized Linear Boltzmann Equation (LBE), in which the Continuous-Energy (CE) fluxes are also integrated over energy bins and transformed into group fluxes. This procedure, based on the Multi-Group (MG) theory, is widely applied in radiation transport and diffusion simulations (Bell and Glasstone, 1970). Careful preparation of MG cross section data, or group constants, is thus a prerequisite for success in acquiring highfidelity LBE solutions. Generation of MG cross section data itself is a complicated multi-step task, since group constants are indispensably coupled with the solution of the LBE, i.e. the flux spectrum in the problem to be solved. Theoretically, a MG cross section library is only valid for the problem that it is built upon. Nevertheless, in practice MG cross section libraries are prepared for similar type of problems, such as thermal reactors, fast reactors, etc., to facilitate the neutronic simulations. Generally, CE point-wise cross section data set, generated by both theoretical models and experimental data, is first converted to Fine-Group (FG) libraries using pre-defined or pre-calculated flux spectra, then, Broad-Group (BG) libraries are collapsed from the fine-group libraries using problem-dependent flux spectra. Proper treatments of energy and spatial self-shielding and resonance cross section are required in the CE-to-MG converting process (Duderstadt and Hamilton, 1976). In this paper, we will focus on the optimization of the fine-to-broad collapsing process, especially on methods to determine the broad optimal group structure.

MG cross section generation techniques are well-established, and implemented in various computer codes, such as NJOY, (MacFarlane et al., 2012), SCALE (ORNL, 2011), and DRAGON (Marleau et al., 1994). MG libraries for different applications are also pre-generated using these computer codes: the VITAMIN-B series of libraries for radiation transport simulations (White et al., 1994), the WIMS-D libraries for thermal reactor applications, (Askew et al., 1966; Leszczynski et al., 2007), the OGIGEN2 library for fast reactor applications (Jin and Oki, 2004), and the KYNEA2 library for Special Nuclear Materials (SNM) detection applications (Varley and Mattingly, 2008), for example. The codes and libraries often utilize the fine-to-broad group collapsing process. While the emphasis in the process is on generation of BG cross section data which maintains the libraries' accuracy for their respective applications on a level close to that of the original FG libraries, there is a paucity of research and method developments on group structure optimization, i.e. how to use fewer number of groups to maintain the same level of accuracy by finding the optimum group structure.

Group structure determination is often based on engineering experience and existing libraries. In a previous study, we have developed a computer code, YGROUP (Yi et al., 2010), for fine-to-broad group cross section collapsing. To maintain high accuracy of the resulting BG libraries, YGROUP provides users several options on collapsing strategies and weighting schemes for group binning and evaluation of BG cross section data for users' specific problems. An approach based on Particle Swarm Optimization (Akbari et al., 2012; Yi and Sjoden, 2013) was applied to automatically determine the optimal group structure. We also developed a hybrid weighting scheme (Yi et al., 2014) to further improve BG library performance for Special Nuclear Materials (SNM) detection applications. 
In this work, we aim to further improve the performance of the heuristic PSO approach with a physics-based Fitness function design based on the contributon transport theory (Painter et al., 1980). In Section 2, we will briefly review on the PSO approach for group structure optimization, and present a Simulation-Driven Fitness Function (SDFF) design with the Dirichlet sampling procedure of the initial group structures. Section 3 will present a detailed derivation of the Physics-Based Fitness Approximation (PBFA) formulation. In Section 4, we will demonstrate that a strong positive correlation exists between SDFF and PBFA through a numerical test on a neutron detector model. Conclusions and future work suggestions will be provided in Section 5.

\section{Group structure heuristic optimization}

We applied one of Swarm Intelligence (SI) algorithms(Beni and Wang, 1993), Particle Swarm Optimization (PSO) (Kennedy and Eberhart, 1995), to the group binning problem: $\mathrm{G} \rightarrow \mathrm{H}$, where $\mathrm{G}$ and $\mathrm{H}$ are the numbers of the fine and broad groups, respectively. One can show that there are a total $\left(\begin{array}{c}\boldsymbol{G}-\mathbf{1} \\ \boldsymbol{H}-\mathbf{1}\end{array}\right)$ number of possible group structures. For example, for collapsing a 100-group library into a 10-group library, the solution space contains $\left(\begin{array}{c}99 \\ 9\end{array}\right) \approx 1.731 \times 10^{12}$ structures. It is not feasible using brute-force approaches to search through the entire solution space.

PSO is a heuristic optimization method, where each solution is represented by a "particle". The search for the optimal solution is performed by random-walking a number of particles in the solution space. The random-walk process is designed to converge particles to the global optimal region in the solution space, while not trapping in local optimums. To efficiently apply PSO to group structure optimization, we built a group graph and converted a group binning problem into a "path search' process (Yi and Sjoden, 2013).

\subsection{Simulation-Driven Fitness Function (SDFF)}

The efficacy of PSO also depends on the design of the fitness function, or the objective function, which is used to evaluate optimality, or fitness, of a solution. For a broad group structure, the straightforward fitness function is to evaluate its optimality by performing transport simulations based on the broad group structure, then comparing the results with the original fine-group structure. Such fitness function design can be called Simulation-driven Fitness Function (SDFF), since timeconsuming simulations are required. For example, we previously used the k-effective difference between BG and FG simulations as the fitness function for a fuel rod model (Yi and Sjoden, 2013). In this section, we will present a more robust SDFF design for detection problems, incorporating both the BG and FG flux and detector response differences. The SDFF design also provides a reference for the discussion in Section 3, where we will derive the Physics-Based Fitness Approximation (PBFA) formulation to avoid the BG transport simulations.

We developed the detection problem SDFF design by considering both the forward and adjoint fluxes differences, as well as the detector response differences. The fitness function is composed of one response term and one flux term, as shown in Eq. 1 


$$
F\left(\Lambda_{H}\right)=w_{0} R_{\text {resp }}+w_{1} R_{\text {flux }}
$$

Where $F\left(\Lambda_{H}\right)$ is the fitness for a given group structure $\Lambda_{H} ; w_{0}$ and $w_{1}$ are two pre-defined and problem-dependent weights. $R_{\text {resp }}$ is the Root-Mean-Square (RMS) of the response relative errors; and $R_{f l u x}$ is the RMS of the flux relative errors. We define $\Lambda_{H}, R_{\text {resp }}$, and $R_{\text {flux }}$ as follows:

$$
\Lambda_{H}=\left\{0, g_{1}^{+}, \ldots g_{H-1}^{+}, G\right\}
$$

$\Lambda_{H}$ is a monotonously increasing integer set with $H+1$ elements, representing the upper group boundaries of a $H-B G$ structure. Note the last $\left(H^{\prime} t h\right)$ element of $\Lambda_{H}$ is $g_{H}^{+}=G$, which is the total number of fine groups, and the zeroth element is set to $g_{0}^{+}=0$. In the same notation, the fine group structure can be expressed as

$$
\Lambda_{G}=\{0,1, \ldots, G-1, G\}
$$

The response difference term $R_{\text {resp }}$ is given as Eq. 4.

$$
R_{\text {resp }}=\sqrt{\frac{1}{2}\left[\left(\frac{R_{B G}-R_{F G}}{R_{F G}}\right)^{2}+\left(\frac{R_{B G}^{*}-R_{F G}^{*}}{R_{F G}^{*}}\right)^{2}\right]}
$$

Where $R_{B G}$ and $R_{F G}$ are the $\mathrm{BG}$ forward response (determined by forward transport simulation using $\Lambda_{H}$ ) and the FG forward response (determined by forward transport simulation using $\Lambda_{G}$ ), respectively. $R_{B G}^{*}$ and $R_{F G}^{*}$ are the BG adjoint response (determined by adjoint transport simulation using $\Lambda_{H}$ ) and the FG adjoint response (determined by adjoint transport simulation using $\Lambda_{G}$ ), respectively (Yi et al., 2012).

The flux difference term $R_{\text {flux }}$ can be evaluated similarly by the following 3 steps:

\section{Step 1. forward flux difference.}

We calculated the RMS of the forward flux difference between the BG and FG transport simulations. Note that the broad group flux in FG simulation can be calculated using Eq. 5.

$$
\phi_{h}^{F G}=\sum_{g=g_{h-1}^{+}+1}^{g_{h}^{+}} \phi_{g}^{F G}
$$

Where $\phi_{g}^{F G}$ is the fine group fluxes acquired from FG transport simulations. $g=g_{h-1}^{+}+1$ to $g=g_{h}^{+}$ are the fine group numbers included in the broad group $h$. Note that we already include a zero zeroth element in $\Lambda_{H}$, so that for $h=1, g=g_{h-1}^{+}+1=g_{0}^{+}+1=1$ is consistent with our notation. Since fluxes are also dependent of positions, and it is not practical to calculate the flux differences for each 
spatial mesh in a problem model, we use the material region averaged fluxes: $\phi_{h, m}^{B G}$ (BG $h$ forward flux evaluated using BG simulations for Material $m$ ), and $\phi_{h, m}^{F G}$ (BG $h$ forward flux evaluated using Eq. 4 and FG simulations). This leads us to rewrite Eq. 5 as Eq. 6.

$$
\phi_{h, m}^{F G}=\sum_{g=g_{h-1}^{+}+1}^{g_{h}^{+}} \phi_{g, m}^{F G} \quad \text { for } m=1,2, \ldots, M
$$

Where $M$ is the total number of materials in the problem model of interest. The RMS of the forward flux relative differences for Material $m$ can be expressed as Eq. 7

$$
R_{m}=\sqrt{\frac{1}{H} \sum_{h=1}^{H}\left(\frac{\phi_{h, m}^{B G}-\phi_{h, m}^{F G}}{\phi_{h, m}^{F G}}\right)^{2}} \quad m=1,2, \ldots, M
$$

The overall forward flux error term $R_{f w d}$ is defined as the weighted RMS of $R_{m}{ }^{\prime} s$

$$
R_{f w d}=\sqrt{\frac{1}{M} \sum_{m=1}^{M}\left(\lambda_{m} R_{m}\right)^{2}}
$$

Where $\lambda_{m}{ }^{\prime} s$ are user-defined material weights. In the YGROUP code (Yi et al., 2010), users can specify $\lambda_{m}$ in the input deck. For example, one option in YGROUP for $\lambda_{m}$ is the corresponding material volume.

\section{Step 2. adjoint flux difference.}

Next, we can use the same approach to evaluate the corresponding overall adjoint flux error term $R_{a d j}$. One should note, instead of the summation mechanism (Eqs. 5 and 6) for BG and FG forward fluxes, the adjoint BG flux is defined as the average of the corresponding FG adjoint fluxes weighted by the corresponding forward fluxes,

$$
\phi_{h, m}^{*, F G}=\sum_{g=g_{h-1}^{+}+1}^{g_{h}^{+}} \frac{\phi_{g, m}^{*, F G} \cdot \phi_{g, m}^{F G}}{\phi_{h, m}^{F G}} \text { for } m=1,2, \ldots, M
$$

Then, $R_{m}^{*}$ and $R_{a d j}$ can be defined by Eq. 10 and 11, respectively.

$$
R_{m}^{*}=\sqrt{\frac{1}{H} \sum_{h=1}^{H}\left(\frac{\phi_{h, m}^{*, B G}-\phi_{h, m}^{*, F G}}{\phi_{h, m}^{*, F G}}\right)^{2}} \quad m=1,2, \ldots, M
$$




$$
R_{a d j}=\sqrt{\frac{1}{M} \sum_{m=1}^{M}\left(\lambda_{m} R_{m}^{*}\right)^{2}} \quad m=1,2, \ldots, M
$$

\section{Step 3. total flux difference.}

Finally, the flux error term $R_{f l u x}$ is the RMS of the forward and adjoint flux RMS's defined by Eqs. 8 and 11.

$$
R_{f l u x}=\sqrt{\frac{R_{f w d}^{2}+R_{a d j}^{2}}{2}}
$$

In summary, the SDFF is evaluated by Eq. 1, and the detector response and flux differences are calculated by Eqs. 4 and 12.

\subsection{Dirichlet distribution sampling}

In PSO, the initial particle positions in the solution space are usually determined by uniform sampling, in hoping that the resulting random walk process would cover most of the space to avoid local extremum traps. In the group structure optimization application, each point in the solution space represents a BG structure. With the help of Eqs. 2 and 3, the initial BG structure sampling process reduces to a problem of uniformly selecting a number of BG structures $\Lambda_{H}{ }^{\prime} s$, which are subsets of $\Lambda_{G}$. It can be proved that the sampling process should follows the parameterized multivariate Dirichlet distribution $D(\alpha)$ (Kotz et al., 2004) with $\alpha=0$.

To demonstrate the initial group structure Dirichlet sampling process, we first define a positive integer set $T_{H}$ to denote the BG size in term of fine groups.

$$
T_{H}=\left\{\tau_{1}, \ldots, \tau_{h}, \ldots, \tau_{H}\right\}
$$

Where $\tau_{h}=g_{h}^{+}-g_{h-1}^{+}$is the size of broad group $h$. Note that $T_{H}$ is the differential set of $\Lambda_{H}$ defined in Eq. 2. One can easily derive one from the other. Let $T_{H}$ be a set of random integer variables with a constraint condition defined by Eq. 14 .

$$
\sum_{h=1}^{H} \tau_{h}=G
$$

We can further transform $T_{H}$ into a set of real random variables normalized by $G$.

$$
X_{H}=\left\{x_{1}, \ldots, x_{h}, \ldots, x_{H}\right\}
$$


Where $x_{h}=\frac{\tau_{h}}{G}$, so that $\sum_{h=1}^{H} x_{h}=1$. And $X_{H}$ is sampled as follows:

First we sample a set of uniformly distributed random variables: $0<\xi_{h}<1$, for $h=1, \ldots, H$. Then, we define the set $Y_{H}$ as Eq. 16.

$$
Y_{H}=\left\{y_{1}, \ldots, y_{h}, \ldots, y_{H}\right\}
$$

Where $y_{h}=-\log \left(\xi_{h}\right), \xi_{h}$ is a random number uniformly distributed in $(0,1) . T_{H}$ can be derived by normalizing $Y_{H}$ using Eq. 17.

$$
X_{H}=\left\{\frac{y_{1}}{y_{t o t}}, \ldots, \frac{y_{h}}{y_{t o t}}, \ldots, \frac{y_{H}}{y_{t o t}}\right\}
$$

Where $y_{\text {tot }}=\sum_{h=1}^{H} y_{h}$.

To convert $X_{H}$ back to $T_{H}$, we use the "round" operation (“closest integer") as Eq. 18.

$$
T_{H}^{\prime}=\left\{\tau_{1}{ }^{\prime}, \ldots, \tau_{h}{ }^{\prime}, \ldots, \tau_{H}{ }^{\prime}\right\} \quad \text { where } \tau_{h}{ }^{\prime}=\operatorname{round}\left(G \cdot x_{h}\right)
$$

Note that due to the "round" operation, the elements of $T^{\prime}{ }_{H}$ (Eq. 18) are not necessarily summed to $G$. So we implement an iterative procedure to adjust $T^{\prime}{ }_{H}$ by increasing the minimum $\tau_{h}^{\prime}$ by one if the summation of $\tau^{\prime}{ }_{h}$ is less than $G$, or decreasing the maximum $\tau^{\prime}{ }_{h}$ by one if the summation is greater than $G$, until that the summation reaches $G$.

\section{Physics-Based Fitness Approximation (PBFA)}

The disadvantage of the PSO approach with SDFF lies in its complexity for users and its computational cost for evaluating the "fitness" of many BG structures along the particle swarm's random walk paths, since "fitness" evaluation often involves a significant number of costly forward and/or adjoint BG transport simulations. For this reason, the PSO approach with SDFF is not ideal to implement in the YGROUP code. Instead, we developed a separate Python code, PyGroup, as a driver to connect the YGROUP and the transport simulation code package TITAN (Yi and Haghighat, 2010).

In this section, we will derive a Physics-Based Fitness Approximation (PBFA) formulation, to avoid the costly transport simulations. Our derivation is based on the contributon transport theory (Painter et al., 1980). In section 3.1, we first derive the Continuous Energy (CE) version of the Contributon Transport Equation (CTE) using the CE forward and adjoint transport equations, then apply the Multi-Group (MG) theory (Duderstadt and Hamilton, 1976) to convert CE-CTE to MG-CTE. In section 3.2, we first apply $\mathrm{MG}$ theory to the forward and adjoint $\mathrm{CE}$ transport equations to acquire the standard MG forward and transport equations, then use them to derive another version of the MGCTE. We will notice a slight difference in the definition of the scattering cross sections in the two 
versions of MG-CTE, which in turn provides us a measuring scheme to gauge the fitness of a BG structure.

\subsection{Multi-group contributon transport equation}

In non-multiplying medium, the time-independent, and Continuous Energy (CE) forward and adjoint transport equations can be written as:

$$
\begin{gathered}
\boldsymbol{\Omega} \cdot \nabla \psi(\boldsymbol{r}, E, \Omega)+\Sigma_{t}(\boldsymbol{r}, E) \psi(\boldsymbol{r}, E, \Omega)=\int_{0}^{\infty} d E^{\prime} \int_{4 \pi} d \Omega^{\prime} \Sigma_{s}\left(\boldsymbol{r}, E^{\prime} \rightarrow E, \Omega^{\prime}\right. \\
\rightarrow \Omega) \psi\left(\boldsymbol{r}, E^{\prime}, \Omega^{\prime}\right)+q(\boldsymbol{r}, E, \Omega) \\
-\boldsymbol{\Omega} \cdot \nabla \psi^{+}(\boldsymbol{r}, E, \Omega)+\Sigma_{t}(\boldsymbol{r}, E) \psi^{+}(\boldsymbol{r}, E, \Omega)=\int_{0}^{\infty} d E^{\prime} \int_{4 \pi} d \Omega^{\prime} \Sigma_{S}\left(\boldsymbol{r}, E \rightarrow E^{\prime}, \Omega\right. \\
\left.\rightarrow \Omega^{\prime}\right) \psi^{+}\left(\boldsymbol{r}, E^{\prime}, \Omega^{\prime}\right)+q^{+}(\boldsymbol{r}, E, \Omega)
\end{gathered}
$$

Here we use the common notation used in (Duderstadt and Hamilton, 1976): $\psi(\boldsymbol{r}, E, \Omega)$ and $\psi^{+}(\boldsymbol{r}, E, \Omega)$ are the neutron angular flux and its adjoint function, respectively. $q(\boldsymbol{r}, E, \Omega)$ and $q^{+}(\boldsymbol{r}, E, \Omega)$ are the external neutron sources and importance sources, respectively. We multiply Eq. 19 by $\psi^{+}(\boldsymbol{r}, E, \Omega)$, and Eq. 20 by $\psi(\boldsymbol{r}, E, \Omega)$, , to acquire Eqs. 21 and 22, respectively.

$$
\begin{aligned}
\psi^{+}(\boldsymbol{r}, E, \Omega) \boldsymbol{\Omega} & \cdot \nabla \psi(\boldsymbol{r}, E, \Omega)+\psi^{+}(\boldsymbol{r}, E, \Omega) \Sigma_{t}(\boldsymbol{r}, E) \psi(\boldsymbol{r}, E, \Omega) \\
& =\psi^{+}(\boldsymbol{r}, E, \Omega) \int_{0}^{\infty} d E^{\prime} \int_{4 \pi} d \Omega^{\prime} \Sigma_{s}\left(\boldsymbol{r}, E^{\prime} \rightarrow E, \Omega^{\prime}\right. \\
& \rightarrow \Omega) \psi\left(\boldsymbol{r}, E^{\prime}, \Omega^{\prime}\right)+\psi^{+}(\boldsymbol{r}, E, \Omega) q(\boldsymbol{r}, E, \Omega) \\
-\psi(\boldsymbol{r}, E, \Omega) \boldsymbol{} \cdot & \nabla \psi^{+}(\boldsymbol{r}, E, \Omega)+\psi(\boldsymbol{r}, E, \Omega) \Sigma_{t}(\boldsymbol{r}, E) \psi^{+}(\boldsymbol{r}, E, \Omega) \\
& =\psi(\boldsymbol{r}, E, \Omega) \int_{0}^{\infty} d E^{\prime} \int_{4 \pi} d \Omega^{\prime} \Sigma_{s}\left(\boldsymbol{r}, E \rightarrow E^{\prime}, \Omega\right. \\
\rightarrow & \left.\Omega^{\prime}\right) \psi^{+}\left(\boldsymbol{r}, E^{\prime}, \Omega^{\prime}\right)+\psi(\boldsymbol{r}, E, \Omega) q^{+}(\boldsymbol{r}, E, \Omega)
\end{aligned}
$$

By substracting Eq. 22 from Eq. 21, the CE contributon transport equation can be written as:

$$
\boldsymbol{\Omega} \cdot \nabla \kappa(\boldsymbol{r}, E, \Omega)=\psi^{+} S-\psi S^{+}+\psi^{+} q-\psi q^{+}
$$

Where $\kappa(\boldsymbol{r}, E, \Omega)=\psi(\boldsymbol{r}, E, \Omega) \psi^{+}(\boldsymbol{r}, E, \Omega)$ is the contributon function. Here we used $S$ and $S^{+}$to denote the scattering source terms in Eqs. 19 and 20, respectively.

$$
S=\int_{0}^{\infty} d E^{\prime} \int_{4 \pi} d \Omega^{\prime} \Sigma_{s}\left(\boldsymbol{r}, E^{\prime} \rightarrow E, \Omega^{\prime} \rightarrow \Omega\right) \psi\left(\boldsymbol{r}, E^{\prime}, \Omega^{\prime}\right)
$$




$$
S^{+}=\int_{0}^{\infty} d E^{\prime} \int_{4 \pi} d \Omega^{\prime} \Sigma_{s}\left(\boldsymbol{r}, E \rightarrow E^{\prime}, \Omega \rightarrow \Omega^{\prime}\right) \psi^{+}\left(\boldsymbol{r}, E^{\prime}, \Omega^{\prime}\right)
$$

By integrating Eq. 23 over Group $g$ energy range $\left(E_{g}^{-}, E_{g}^{+}\right)$, we can derive the MG form of the contributon equation.

$$
\begin{aligned}
\boldsymbol{\Omega} \cdot \nabla \kappa_{\mathrm{g}}(\boldsymbol{r}, \Omega) & =\int_{4 \pi} d \Omega^{\prime}\left\{\psi_{g}^{+}(\boldsymbol{r}, \Omega) \sum_{g^{\prime}=1}^{G} \psi_{g^{\prime}}\left(\boldsymbol{r}, \Omega^{\prime}\right) \Sigma_{s, g^{\prime} \rightarrow g}^{c}\left(\boldsymbol{r}, \Omega^{\prime} \rightarrow \Omega\right)\right. \\
& \left.-\psi_{g}(\boldsymbol{r}, \Omega) \sum_{g^{\prime}=1}^{G} \psi_{g^{\prime}}^{+}\left(\boldsymbol{r}, \Omega^{\prime}\right) \Sigma_{s, g \rightarrow g^{\prime}}^{c}\left(\boldsymbol{r}, \Omega \rightarrow \Omega^{\prime}\right)\right\}+\psi_{g}^{+} q_{g}-\psi_{g} q_{g}^{+}
\end{aligned}
$$

In Eq. 26, we denote the MG versions of angular flux, adjoint function and contributon function as $\psi_{g}(\boldsymbol{r}, \Omega), \psi_{g}^{+}(\boldsymbol{r}, \Omega)$, and $\kappa_{\mathrm{g}}(\boldsymbol{r}, \Omega)$. They are defined as Eqs. 27-29.

$$
\begin{gathered}
\psi_{g}(\boldsymbol{r}, \Omega)=\int_{E_{g}^{-}}^{E_{g}^{+}} d E \psi(\boldsymbol{r}, E, \Omega) \\
\psi_{g}^{+}(\boldsymbol{r}, \Omega)=\frac{\int_{E_{g}^{-}}^{E^{+}} d E \psi(\boldsymbol{r}, E, \Omega) \psi^{+}(\boldsymbol{r}, E, \Omega)}{\psi_{g}(\boldsymbol{r}, \Omega)} \\
\kappa_{\mathrm{g}}(\boldsymbol{r}, \Omega)=\int_{E_{g}^{-}}^{E_{g}^{-}} d E \kappa\left(\boldsymbol{r}, E^{\prime}, \Omega\right)=\int_{E_{g}^{-}}^{E_{g}^{+}} d E \psi(\boldsymbol{r}, E, \Omega) \psi^{+}(\boldsymbol{r}, E, \Omega)=\psi_{g}(\boldsymbol{r}, \Omega) \psi_{g}^{+}(\boldsymbol{r}, \Omega)
\end{gathered}
$$

By comparing Eq. 26 with the CE version (Eq. 23), the group-to-group scattering cross section $\Sigma_{s, g^{\prime} \rightarrow g}^{c}\left(\boldsymbol{r}, \Omega^{\prime} \rightarrow \Omega\right)$ and $\Sigma_{s, g^{\prime} \rightarrow g}^{c}\left(\boldsymbol{r}, \Omega^{\prime} \rightarrow \Omega\right)$ in Eq. 26 can be written as:

$$
\begin{aligned}
& \sum_{s, g^{\prime} \rightarrow g}^{c}\left(\boldsymbol{r}, \Omega^{\prime} \rightarrow \Omega\right)=\frac{\int_{E_{g^{\prime}}^{-}}^{E_{g^{\prime}}^{+}} d E^{\prime} \int_{E_{g}^{-}}^{E_{g}^{+}} d E \psi\left(\boldsymbol{r}, E^{\prime}, \Omega^{\prime}\right) \Sigma_{s}\left(\boldsymbol{r}, E^{\prime} \rightarrow E, \Omega^{\prime} \rightarrow \Omega\right) \psi^{+}(\boldsymbol{r}, E, \Omega)}{\psi_{g^{\prime}}\left(\boldsymbol{r}, \Omega^{\prime}\right) \psi_{g}^{+}(\boldsymbol{r}, \Omega)} \\
& \sum_{s, g \rightarrow g^{\prime}}^{c}\left(\boldsymbol{r}, \Omega \rightarrow \Omega^{\prime}\right)=\frac{\int_{E_{g^{\prime}}^{-}}^{E_{g^{\prime}}^{+}} d E^{\prime} \int_{E_{g}^{-}}^{E_{g}^{+}} d E \psi(\boldsymbol{r}, E, \Omega) \Sigma_{s}\left(\boldsymbol{r}, E \rightarrow E^{\prime}, \Omega \rightarrow \Omega^{\prime}\right) \psi^{+}\left(\boldsymbol{r}, E^{\prime}, \Omega^{\prime}\right)}{\psi_{g}(\boldsymbol{r}, \Omega) \psi_{g^{\prime}}^{+}\left(\boldsymbol{r}, \Omega^{\prime}\right)}
\end{aligned}
$$

Note that Eq. 30 can also be acquired by switching $g \Leftrightarrow g^{\prime}$ and $\Omega \Leftrightarrow \Omega^{\prime}$ in Eq. 31. Therefore, the definition of the MG scattering cross section by Eq. 30 is mathematically consistent. Physically, the conservation quantity in the CE-to-MG transform is the total neutron importance, which becomes more evident by rewriting Eq. 30 as Eq. 32:

$$
\begin{gathered}
\psi_{g^{\prime}}\left(\boldsymbol{r}, \Omega^{\prime}\right) \Sigma_{s, g^{\prime} \rightarrow g}^{c}\left(\boldsymbol{r}, \Omega^{\prime} \rightarrow \Omega\right) \psi_{g}^{+}(\boldsymbol{r}, \Omega)=\int_{E_{g^{\prime}}^{-}}^{E_{g^{\prime}}^{+}} d E^{\prime} \int_{E_{g}^{-}}^{E_{g}^{+}} d E \psi\left(\boldsymbol{r}, E^{\prime}, \Omega^{\prime}\right) \Sigma_{s}\left(\boldsymbol{r}, E^{\prime}\right. \\
\left.\rightarrow E, \Omega^{\prime} \rightarrow \Omega\right) \psi^{+}(\boldsymbol{r}, E, \Omega)
\end{gathered}
$$


Eq. 32 shows that the total amount of "importance" carried by the outgoing neutrons remains the same using either the MG or the CE formulation, which is the basis of the MG scattering cross section defined in Eq. 30. We need to emphasize Eq. 30 is essentially the same approach as the Hybrid Weighting Scheme (HWS) (Yi et al., 2014). We previously used HWS as a formulation for collapsing FG to BG cross section library, while here Eq. 30 is used for converting CE to MG cross section data. Both approaches share the same basis that the "importance" is conserved after the conversion.

\subsection{PBFA formulation}

We now present an alternative derivation of the contributon transport equation. Instead of the CE forward and adjoint transport equations, we start with their corresponding MG versions.

$$
\begin{aligned}
\boldsymbol{\Omega} \cdot \nabla \psi_{\mathrm{g}}(\boldsymbol{r}, \Omega) & +\sum_{t, g}(\boldsymbol{r}) \psi_{\mathrm{g}}(\boldsymbol{r}, \Omega) \\
& =\sum_{g^{\prime}=1}^{G} \int_{4 \pi} d \Omega^{\prime} \Sigma_{s, g^{\prime} \rightarrow g}\left(\boldsymbol{r}, \Omega^{\prime} \rightarrow \Omega\right) \psi_{g^{\prime}}\left(\boldsymbol{r}, \Omega^{\prime}\right)+q_{g}(\boldsymbol{r}, \Omega) \\
-\boldsymbol{\Omega} \cdot \nabla \psi_{g}^{+}(\boldsymbol{r}, \Omega) & +\sum_{t, g}(\boldsymbol{r}) \psi^{+}(\boldsymbol{r}, E, \Omega) \\
& =\sum_{g^{\prime}=1}^{G} \int_{4 \pi} d \Omega^{\prime} \Sigma_{s, g \rightarrow g^{\prime}}\left(\boldsymbol{r}, \Omega \rightarrow \Omega^{\prime}\right) \psi_{g^{\prime}}^{+}\left(\boldsymbol{r}, \Omega^{\prime}\right)+q_{g}^{+}(\boldsymbol{r}, \Omega)
\end{aligned}
$$

Eq. 33 can be derived by integrating Eq. 19 over Group g energy range $\left(E_{g}^{-}, E_{g}^{+}\right)$. And in the process, the group flux and group constants can be defined by conserving the corresponding reaction rates (Duderstadt and Hamilton, 1976). However, Eq. 34 cannot be derived by integrating Eq. 20, because such integration would lead to a conflictive definition of the total cross section, which is weighted by the adjoint function, instead of the forward flux. We note that Eq. 34 is derived by using the mathematical properties of the adjoint operator and the requirement of the vacuum boundary condition (Bell and Glasstone, 1970). Nevertheless, we can acquire the contributon transport equation by multiplying Eq. 33 by $\psi_{g}^{+}(\boldsymbol{r}, \Omega)$, and Eq. 34 by $\psi_{\mathrm{g}}(\boldsymbol{r}, \Omega)$, then subtracting the resulting two equations.

$$
\begin{aligned}
\boldsymbol{\Omega} \cdot \nabla \kappa_{\mathrm{g}}(\boldsymbol{r}, \Omega) & =\int_{4 \pi} d \Omega^{\prime}\left\{\psi_{g}^{+}(\boldsymbol{r}, \Omega) \sum_{g^{\prime}=1}^{G} \psi_{g^{\prime}}(\boldsymbol{r}, \Omega) \Sigma_{s, g^{\prime} \rightarrow g}\left(\boldsymbol{r}, \Omega^{\prime} \rightarrow \Omega\right)\right. \\
& \left.-\psi_{g}(\boldsymbol{r}, \Omega) \sum_{g^{\prime}=1}^{G} \psi_{g^{\prime}}^{+}(\boldsymbol{r}, \Omega) \Sigma_{s, g \rightarrow g^{\prime}}\left(\boldsymbol{r}, \Omega \rightarrow \Omega^{\prime}\right)\right\}+\psi_{g}^{+} q_{g}-\psi_{g} q_{g}^{+}
\end{aligned}
$$

Where $\psi_{g}(\boldsymbol{r}, \Omega), \psi_{g}^{+}(\boldsymbol{r}, \Omega)$, and $\kappa_{\mathrm{g}}(\boldsymbol{r}, \Omega)$ are the same as defined in Eqs. 27-29. The scattering cross section, however, is different. 


$$
\Sigma_{s, g^{\prime} \rightarrow g}\left(\boldsymbol{r}, \Omega^{\prime} \rightarrow \Omega\right)=\frac{\int_{E_{g^{\prime}}}^{E_{g^{\prime}}^{+}} d E^{\prime} \int_{E_{g}^{-}}^{E_{g}^{+}} d E \psi\left(\boldsymbol{r}, E^{\prime}, \Omega\right) \Sigma_{s}\left(\boldsymbol{r}, E^{\prime} \rightarrow E, \Omega^{\prime} \rightarrow \Omega\right)}{\psi_{g^{\prime}}(\boldsymbol{r}, \Omega)}
$$

Eq. 36 is the flux-weighting group constant generation formulation, where the corresponding reaction rates are conserved. The scattering terms in the two versions of MG-CTE (Eqs. 23 and 35) differ only on the scattering cross section, defined by Eqs. 30 and 36, respectively. While Eq. 30 is widely used to conserve the total reaction rates, Eq. 36, where the conserved quantity is the total neutron importance, could be more efficient in some cases as we demonstrated previously (Yi et al., 2014). The contributon transport equation itself has very interesting physical meanings that lead to some useful applications (Painter et al., 1980). For example, the solution of the contributon equation in a source-detector model will highlight the optimal path for neutrons traveling from the source to the detector region. Therefore, it provides effective variance reduction guidelines for Monte Carlo simulations.

We note that both Eqs. 30 and 36 will approach the CE scattering cross section as the number of energy groups approaches infinity, i.e. the group energy range approaches zero.

$$
\begin{gathered}
\Sigma_{s, g^{\prime} \rightarrow g}^{c}\left(\boldsymbol{r}, \Omega^{\prime} \rightarrow \Omega\right) \rightarrow \Sigma_{s}\left(\boldsymbol{r}, E^{\prime} \rightarrow E, \Omega^{\prime} \rightarrow \Omega\right) ; \\
\Sigma_{s, g^{\prime} \rightarrow g}\left(\boldsymbol{r}, \Omega^{\prime} \rightarrow \Omega\right) \rightarrow \Sigma_{s}\left(\boldsymbol{r}, E^{\prime} \rightarrow E, \Omega^{\prime} \rightarrow \Omega\right) ; \\
\text { for } E^{\prime} \in\left(E_{g^{\prime}}^{-}, E_{g^{\prime}}^{+}\right), \quad E \in\left(E_{g}^{-}, E_{g}^{+}\right), \\
\text {as }\left(E_{g^{\prime}}^{+}-E_{g^{\prime}}^{-}\right) \rightarrow 0 ;\left(E_{g}^{+}-E_{g}^{-}\right) \rightarrow 0
\end{gathered}
$$

Eq. 37 indicates the difference in the scattering cross section formulations (Eqs. 30 and 36) is introduced by the discretization of the energy domain, i.e. the multigroup theory. On the other hand, we could use this difference as a metric to evaluate the performance a CE-to-MG library conversion process. A group structure that minimizes this difference could indicate that the corresponding group structure is an optimal one.

In our application, we are actually interested in the FG-to-BG library conversion. Therefore, we recast Eqs. 30 and 36 to the FG-to-BG formulations.

$$
\begin{gathered}
\sum_{s, h^{\prime} \rightarrow h}^{c}\left(\boldsymbol{r}, \Omega^{\prime} \rightarrow \Omega\right)=\frac{\sum_{g^{\prime}=g_{h^{\prime}}^{-}}^{g_{h^{\prime}}^{+}} \sum_{g=g_{h}^{-}}^{g_{h}^{+}} \psi_{g^{\prime}}\left(\boldsymbol{r}, \Omega^{\prime}\right) \Sigma_{s, g^{\prime} \rightarrow g}\left(\boldsymbol{r}, \Omega^{\prime} \rightarrow \Omega\right) \psi_{g}^{+}(\boldsymbol{r}, \Omega)}{\psi_{h^{\prime}}\left(\boldsymbol{r}, \Omega^{\prime}\right) \psi_{h}^{+}(\boldsymbol{r}, \Omega)} \\
\sum_{s, h^{\prime} \rightarrow h}\left(\boldsymbol{r}, \Omega^{\prime} \rightarrow \Omega\right)=\frac{\sum_{g^{\prime}=g_{h^{\prime}}^{-}}^{g_{\prime^{\prime}}^{+}} \sum_{g=g_{h}^{-}}^{g_{h}^{+}} \psi_{g^{\prime}}\left(\boldsymbol{r}, \Omega^{\prime}\right) \Sigma_{s, g^{\prime} \rightarrow g}\left(\boldsymbol{r}, \Omega^{\prime} \rightarrow \Omega\right)}{\psi_{h^{\prime}}\left(\boldsymbol{r}, \Omega^{\prime}\right)}
\end{gathered}
$$

Where we used our previous notation on FG and FG groups: $g=1,2, \ldots, G$ and $h=1,2, \ldots, H$; and G and $\mathrm{H}$ are the total numbers of fine groups and broad groups, respectively. Eqs. 38 and 39 are the FGto-BG versions of Eqs. 30 and 36, respectively. They can be derived by replacing the energy 
integration operations, $\int_{E_{g}^{-}}^{E_{g}^{+}} d E$ and $\int_{E_{g^{\prime}}}^{E_{g^{\prime}}^{+}} d E^{\prime}$, to corresponding group summation operations, $\sum_{g=g_{h}^{-}}^{g_{h}^{+}}$, and $\sum_{g^{\prime}=g_{h \prime}^{-}}^{g_{h \prime}^{+}}$. Note we also derived Eq. 38 in the matrix form as the Hybrid Weighting Scheme (Yi et al., 2014). Here we demonstrated that the same formulation can by acquired using the contributon transport equation. Similar to Eq. 37, we can show that both $\Sigma_{s, h \prime \rightarrow h}^{c}\left(\boldsymbol{r}, \Omega^{\prime} \rightarrow \Omega\right)$ and $\Sigma_{s, h \rightarrow h}\left(\boldsymbol{r}, \Omega^{\prime} \rightarrow\right.$ $\Omega$ ) will be equal to the exact $\Sigma_{s, g^{\prime} \rightarrow g}\left(\boldsymbol{r}, \Omega^{\prime} \rightarrow \Omega\right.$ ), if the broad group structure is the same as the original fine group structure, i.e. $G=H$. To complete Eqs. 35, 38 and 39, we also need to define the corresponding broad group flux, adjoint function, and contributon function.

$$
\begin{aligned}
& \psi_{h}(\boldsymbol{r}, \Omega)=\sum_{g=g_{h}^{-}}^{g_{h}^{+}} \psi_{g}(\boldsymbol{r}, \Omega) \\
& \psi_{h}^{+}(\boldsymbol{r}, \Omega)=\frac{\sum_{g=g_{h}^{-}}^{g_{h}^{+}} \psi_{g}(\boldsymbol{r}, \Omega) \psi_{g}^{+}(\boldsymbol{r}, \Omega)}{\sum_{g=g_{h}^{-}}^{g_{h}^{+}} \psi_{g}(\boldsymbol{r}, \Omega)} \\
& \kappa_{h}(\boldsymbol{r}, \Omega)=\sum_{g=g_{h}^{-}}^{g_{h}^{+}} \kappa_{g}(\boldsymbol{r}, \Omega)=\sum_{g=g_{h}^{-}}^{g_{h}^{+}} \psi_{g}(\boldsymbol{r}, \Omega) \psi_{g}^{+}(\boldsymbol{r}, \Omega)=\psi_{h}(\boldsymbol{r}, \Omega) \psi_{h}^{+}(\boldsymbol{r}, \Omega)
\end{aligned}
$$

Finally, we define a fitness function based on the scattering cross section difference, bi-linearly weighted by the incoming BG flux and outgoing BG adjoint function.

$$
F_{a}\left(\Lambda_{H}\right)=\sum_{h=1}^{H} \sum_{h^{\prime}=1}^{H} \psi_{h}(\boldsymbol{r}, \Omega)\left|\Sigma_{s, h^{\prime} \rightarrow h}^{c}\left(\boldsymbol{r}, \Omega^{\prime} \rightarrow \Omega\right)-\Sigma_{s, h^{\prime} \rightarrow h}\left(\boldsymbol{r}, \Omega^{\prime} \rightarrow \Omega\right)\right| \psi_{h^{\prime}}^{+}(\boldsymbol{r}, \Omega)
$$

Where we use the previous notation $\Lambda_{H}=\left\{0, g_{1}^{+}, \ldots g_{H-1}^{+}, G\right\}$ to represent a broad group structure. Comparing with the Simulation-Driven Fitness Function (SDFF) (Eq. 1), which we can regard as the true "fitness" of a BG structure, Eq. 43 is a Physics-Based Fitness Approximation (PBFA), since its values are not guaranteed to always behave the same as SDFF. However, the advantage of PBFA is that it can be evaluated without performing BG transport simulations. Given a BG structure, all terms on the right hand side of Eq. 43, including the incoming BG flux, the BG scattering cross section difference, and the outgoing BG adjoint function, can be calculated using the reference FG forward and adjoint simulation results. Therefore, it has the potential to reduce the PSO computation cost significantly. Eq. 43 also has dependencies on both space and angle. In practice, instead of angular flux for each mesh, we will use averaged scalar fluxes for each material region weighted by the corresponding material volume.

\section{Numerical results}

A new PBFA module is developed in the YGROUP code, while the SDFF is implemented in the aforementioned PyGroup code, a Python/Numpy/Scipy driver script using the PSO algorithm, in which SDFF is evaluated by the TITAN transport code, and BG cross section data is collapsed by the 
YGROUP code. The Dirichlet distribution sampling is applied in both PyGroup and YGROUP. To test the performance of PBFA, we applied both YGROUP and PyGroup codes to the Dual Range Coincidence Counter (DRCC) model (Ensslin et al., 1978; Reilly et al., 1991).

DRCC is an established detector design which can be used for Plutonium scrap detection. Figure 1 shows the cross sectional area of DRCC. The detector consists of $24 \mathrm{He}-3$ tubes of 2.54-cm diameter filled to a pressure of $4 \mathrm{~atm}$. The inner and outer polyethylene cylinders (moderators) are each $3.0 \mathrm{~cm}$ thick. A dual-range neutron assay capability is achieved in the DRCC design by having two removable cadmium sleeves near the ${ }^{3} \mathrm{He}$ detectors. The cadmium sleeve $(1.0 \mathrm{~mm}$ thick) on the inside of the well stops low-energy neutrons from returning to the sample position, thereby reducing multiplication for high-mass loadings.

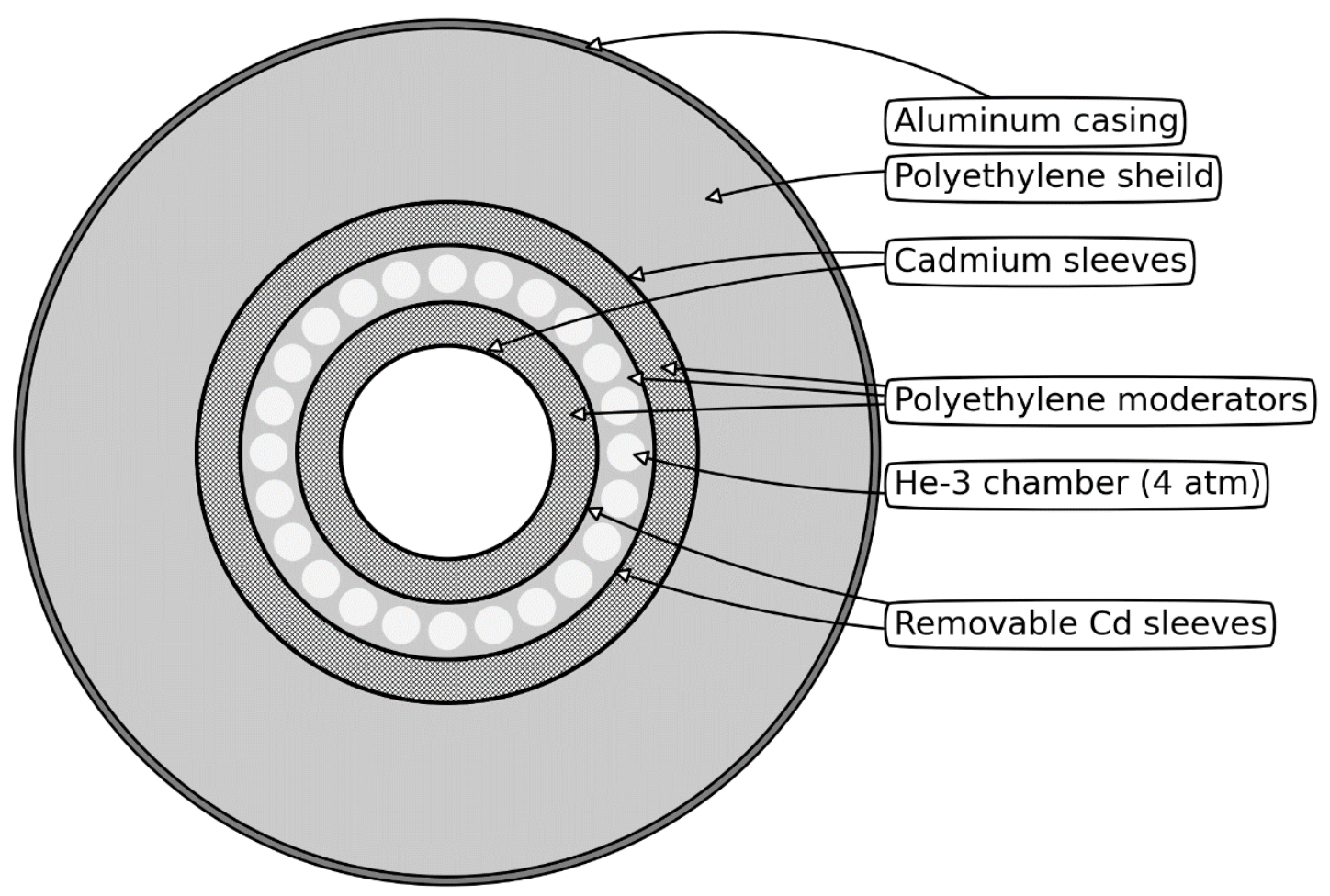

Figure 1. Dual-Range Coincidence Counter cross-sectional view.

\subsection{PyGroup/SDFF results}

We built a modified 3-D DRCC model composed of 3 z-levels, with 4 coarse meshes per z-level, and total 145,152 fine meshes. Five materials (Polyethylene, aluminum, He-3, water and air) are used in the 30-FG library we generated for detector efficiency optimization applications (Yi et al., 2012). The forward and adjoint transport simulations in PyGroup are performed by the TITAN code using $\mathrm{S}_{16}$ Level Symmetric quadrature. The forward source, located in the center of DRCC, is a SNM solution sample with $\mathrm{Pu}-239$ spontaneous fission spectrum, while the adjoint source is located in the He-3 chambers with He-3 absorption cross section as source spectrum. PyGroup/SDFF collapsed the 30-FG library into an optimal 3-BG library. Table 1 shows the optimal 3-BG structure and its fitness metrics.

Table 1. Fitness metrics of the optimal 3-BG structure for the 3-D DRCC model. 


\begin{tabular}{lcccc} 
Overall fitness: & \multicolumn{5}{c}{0.0559} \\
Response Rel. Err.(RMS fwd adj) : & 0.0554 & 0.0556 & 0.0551 \\
Flux Rel. Err. (RMS fwd adj) : & 0.0587 & 0.058 & 0.0593 \\
\hline Forward Flux Rel. Err: Material Name:(RMS, BG number) \\
Mat. Name & RMS & BG_1 & BG_2 & BG_3 \\
Polyethylene & $6.75 \%$ & $10.27 \%$ & $3.40 \%$ & $4.43 \%$ \\
Al & $4.20 \%$ & $7.01 \%$ & $1.83 \%$ & $0.69 \%$ \\
he3-4atm & $6.91 \%$ & $9.77 \%$ & $0.52 \%$ & $6.90 \%$ \\
Water & $6.60 \%$ & $1.72 \%$ & $5.82 \%$ & $9.67 \%$ \\
Air-humid & $3.67 \%$ & $4.16 \%$ & $0.89 \%$ & $4.73 \%$ \\
\hline Adjoint Flux Rel. Err: & Material Name:(RMS, BG number) \\
Mat. Name & RMS & BG_1 & BG_2 & BG_3 \\
Polyethylene & $5.74 \%$ & $5.52 \%$ & $6.09 \%$ & $5.61 \%$ \\
Al & $7.15 \%$ & $6.14 \%$ & $6.14 \%$ & $8.84 \%$ \\
he3-4atm & $2.32 \%$ & $2.20 \%$ & $1.44 \%$ & $3.03 \%$ \\
Water & $6.80 \%$ & $3.18 \%$ & $0.18 \%$ & $11.33 \%$ \\
Air-humid & $6.36 \%$ & $4.68 \%$ & $4.70 \%$ & $8.79 \%$ \\
\hline
\end{tabular}

PyGroup identified the optimal structure, 1-7, 8-12, 13-30, with an overall fitness value 0.0559. Both response and flux errors relative to the 30-FG reference results are taken into account in calculating the fitness value. The detector responses calculated by using the optimal 3-BG library agrees well with the $30-\mathrm{FG}$ library with a relative difference $\sim 5 \%$. Table 1 also shows the detailed forward and adjoint flux differences for the 5 materials in the DRCC model. These metrics are defined in the Section 2.1. Note that the BG reference fluxes are calculated using Eqs. 40 and 41 based on the 30-FG forward and adjoint transport simulation results.

Table 2. 3-BG structure fitness dictionary for 3-D DRCC model

\begin{tabular}{|c|c|c|c|c|c|c|c|c|c|}
\hline$\#$ & BG structure & Fitness & Resp. RMS & Flux. RMS & $\#$ & BG structure & Fitness & Resp RMS & Flux RMS \\
\hline 1 & 1-7 8-8 9-30 & 0.088218 & 0.09221 & 0.065596 & 56 & $1-34-56-30$ & 0.227412 & 0.249021 & 0.104959 \\
\hline 2 & $1-14 \quad 15-16 \quad 17-30$ & 0.254906 & 0.25681 & 0.244122 & 57 & $1-910-2122-30$ & 0.14024 & 0.124832 & 0.227555 \\
\hline 3 & $1-12-17 \quad 18-30$ & 0.322871 & 0.343598 & 0.205418 & 58 & $1-14 \quad 15-27 \quad 28-30$ & 0.274483 & 0.26814 & 0.310428 \\
\hline 4 & $1-12-2021-30$ & 0.432369 & 0.441944 & 0.378109 & 59 & $1-13$ 14-18 19-30 & 0.24431 & 0.248774 & 0.219015 \\
\hline 5 & $1-56-22 \quad 23-30$ & 0.541361 & 0.552695 & 0.47714 & 60 & $1-56-67-30$ & 0.17146 & 0.185822 & 0.090073 \\
\hline 6 & $1-89-9$ 10-30 & 0.103529 & 0.103169 & 0.105573 & 61 & $1-18$ 19-19 20-30 & 0.36334 & 0.371406 & 0.31763 \\
\hline 7 & $1-23-18 \quad 19-30$ & 0.35402 & 0.375762 & 0.230813 & 62 & $1-89-22 \quad 23-30$ & 0.139573 & 0.117059 & 0.267154 \\
\hline 8 & $1-11 \quad 12-22 \quad 23-30$ & 0.188027 & 0.17994 & 0.233857 & 63 & $1-12 \quad 13-27 \quad 28-30$ & 0.248991 & 0.234906 & 0.328804 \\
\hline 9 & $1-12-1213-30$ & 0.225421 & 0.234461 & 0.174194 & 64 & $1-12-56-30$ & 0.226075 & 0.246867 & 0.108254 \\
\hline 10 & $1-10 \quad 11-1920-30$ & 0.132697 & 0.123233 & 0.186327 & 65 & $1-23-2122-30$ & 0.471511 & 0.461266 & 0.529568 \\
\hline 11 & $1-89-2425-30$ & 0.153472 & 0.116182 & 0.364779 & 66 & $1-22 \quad 23-2324-30$ & 0.531616 & 0.466228 & 0.902147 \\
\hline 12 & $1-89-2122-30$ & 0.132637 & 0.116102 & 0.226337 & 67 & $1-16$ 17-17 18-30 & 0.292145 & 0.298495 & 0.256159 \\
\hline 13 & $1-89-17 \quad 18-30$ & 0.082353 & 0.073863 & 0.130463 & 68 & $1-2425-27 \quad 28-30$ & 0.604444 & 0.464519 & 1.397355 \\
\hline 14 & $1-11 \quad 12-12 \quad 13-30$ & 0.158053 & 0.149409 & 0.207031 & 69 & $1-1920-2526-30$ & 0.410926 & 0.403647 & 0.452175 \\
\hline 15 & $1-56-16 \quad 17-30$ & 0.14051 & 0.148004 & 0.098043 & 70 & $1-56-15 \quad 16-30$ & 0.131556 & 0.138683 & 0.091173 \\
\hline 16 & $1-34-45-30$ & 0.253203 & 0.277677 & 0.114518 & 71 & $1-11 \quad 12-15 \quad 16-30$ & 0.149015 & 0.138884 & 0.206424 \\
\hline 17 & $1-67-78-30$ & 0.11678 & 0.124383 & 0.073694 & 72 & $1-34-67-30$ & 0.172577 & 0.18809 & 0.084669 \\
\hline 18 & $1-11 \quad 12-18 \quad 19-30$ & 0.16177 & 0.154819 & 0.201159 & 73 & $1-10 \quad 11-16 \quad 17-30$ & 0.105179 & 0.091588 & 0.182195 \\
\hline 19 & $1-2122-2425-30$ & 0.500718 & 0.461089 & 0.725279 & 74 & $1-23-45-30$ & 0.25195 & 0.275575 & 0.118073 \\
\hline 20 & $1-45-56-30$ & 0.226444 & 0.247104 & 0.109371 & 75 & $1-12-89-30$ & 0.114001 & 0.118394 & 0.089109 \\
\hline 21 & $1-12-2425-30$ & 0.535004 & 0.464277 & 0.935793 & 76 & $1-13 \quad 14-25 \quad 26-30$ & 0.266265 & 0.259955 & 0.302024 \\
\hline 22 & $1-9 \quad 10-12 \quad 13-30$ & 0.080037 & 0.068464 & 0.145619 & 77 & $1-14 \quad 15-18 \quad 19-30$ & 0.252596 & 0.257989 & 0.222032 \\
\hline
\end{tabular}




\begin{tabular}{|c|c|c|c|c|c|c|c|c|c|}
\hline 23 & $1-78-11 \quad 12-30$ & 0.060231 & 0.061256 & 0.054422 & 78 & $1-67-14 \quad 15-30$ & 0.088729 & 0.093202 & 0.063387 \\
\hline 24 & $1-2 \quad 3-13 \quad 14-30$ & 0.246806 & 0.258676 & 0.17954 & 79 & $1-8 \quad 9-13 \quad 14-30$ & 0.065483 & 0.055945 & 0.119528 \\
\hline 25 & 1-9 $10-13 \quad 14-30$ & 0.079498 & 0.067328 & 0.148465 & 80 & $1-2324-24 \quad 25-30$ & 0.568646 & 0.466505 & 1.147447 \\
\hline 26 & $1-10$ 11-18 $19-30$ & 0.124738 & 0.114416 & 0.183234 & 81 & $1-23-34-30$ & 0.350351 & 0.382025 & 0.170866 \\
\hline 27 & $1-11 \quad 12-25 \quad 26-30$ & 0.215362 & 0.186538 & 0.378694 & 82 & $1-12-78-30$ & 0.118526 & 0.126859 & 0.07131 \\
\hline 28 & $1-12 \quad 13-13 \quad 14-30$ & 0.221797 & 0.220394 & 0.229747 & 83 & $1-89-15 \quad 16-30$ & 0.06729 & 0.057465 & 0.122967 \\
\hline 29 & $1-78-1920-30$ & 0.10606 & 0.103391 & 0.121179 & 84 & $1-17 \quad 18-18 \quad 19-30$ & 0.328045 & 0.337849 & 0.272487 \\
\hline 30 & $1-12-23-30$ & 0.416435 & 0.453246 & 0.207835 & 85 & $1-12 \quad 13-22 \quad 23-30$ & 0.231981 & 0.232456 & 0.229287 \\
\hline 31 & $1-12-1920-30$ & 0.383129 & 0.403415 & 0.268177 & 86 & $1-34-1920-30$ & 0.318865 & 0.333852 & 0.23394 \\
\hline 32 & $1-7 \quad 8-13 \quad 14-30$ & 0.05754 & 0.056807 & 0.061691 & 87 & $1-34-10 \quad 11-30$ & 0.133593 & 0.13977 & 0.098595 \\
\hline 33 & $1-12-910-30$ & 0.12583 & 0.128938 & 0.108217 & 88 & $1-23-89-30$ & 0.113885 & 0.118402 & 0.088291 \\
\hline 34 & $1-67-13 \quad 14-30$ & 0.086611 & 0.090994 & 0.061777 & 89 & 1-7 8-12 13-30 & 0.055855 & 0.055361 & 0.058654 \\
\hline 35 & $1-910-16 \quad 17-30$ & 0.083417 & 0.070917 & 0.154251 & 90 & $1-5 \quad 6-13 \quad 14-30$ & 0.122806 & 0.129564 & 0.084506 \\
\hline 36 & $1-15 \quad 16-2021-30$ & 0.272758 & 0.280906 & 0.226585 & 91 & $1-12$ 13-18 $19-30$ & 0.21664 & 0.217516 & 0.211678 \\
\hline 37 & $1-2021-2122-30$ & 0.449089 & 0.437265 & 0.51609 & 92 & $1-7 \quad 8-18 \quad 19-30$ & 0.093638 & 0.092577 & 0.09965 \\
\hline 38 & $1-12-67-30$ & 0.170084 & 0.184621 & 0.087707 & 93 & $1-56-12 \quad 13-30$ & 0.114135 & 0.120421 & 0.078514 \\
\hline 39 & $\begin{array}{llll}1-15 & 16-18 & 19-30\end{array}$ & 0.269163 & 0.276232 & 0.229105 & 94 & $1-56-89-30$ & 0.101836 & 0.109576 & 0.057979 \\
\hline 40 & $1-9$ 10-14 15-30 & 0.079747 & 0.067429 & 0.149549 & 95 & $1-15$ 16-16 $17-30$ & 0.27407 & 0.276838 & 0.258386 \\
\hline 41 & $1-12-10 \quad 11-30$ & 0.148389 & 0.151105 & 0.132995 & 96 & $1-12 \quad 13-1920-30$ & 0.219602 & 0.221235 & 0.210348 \\
\hline 42 & $1-7 \quad 8-9$ 10-30 & 0.080527 & 0.084188 & 0.059781 & 97 & $1-8 \quad 9-10 \quad 11-30$ & 0.091174 & 0.088596 & 0.105784 \\
\hline 43 & $1-11 \quad 12-2829-30$ & 0.2193 & 0.186559 & 0.404833 & 98 & $1-12 \quad 13-15 \quad 16-30$ & 0.215672 & 0.213747 & 0.226576 \\
\hline 44 & $1-12-45-30$ & 0.251999 & 0.275755 & 0.117382 & 99 & $1-89-16 \quad 17-30$ & 0.070391 & 0.060578 & 0.125994 \\
\hline 45 & $1-45-16 \quad 17-30$ & 0.1602 & 0.169042 & 0.110097 & 100 & $1-89-14 \quad 15-30$ & 0.065952 & 0.056295 & 0.120676 \\
\hline 46 & $1-7 \quad 8-14 \quad 15-30$ & 0.058513 & 0.057748 & 0.062854 & 101 & $1-67-1112-30$ & 0.078277 & 0.083329 & 0.04965 \\
\hline 47 & $1-14 \quad 15-2930-30$ & 0.279819 & 0.268077 & 0.346356 & 102 & $1-2021-2930-30$ & 0.469798 & 0.442204 & 0.626162 \\
\hline 48 & $1-14 \quad 15-15 \quad 16-30$ & 0.259876 & 0.261501 & 0.250667 & 103 & $1-56-1920-30$ & 0.202805 & 0.20868 & 0.169511 \\
\hline 49 & $1-67-12 \quad 13-30$ & 0.081956 & 0.086356 & 0.057025 & 104 & $1-45-22 \quad 23-30$ & 0.304721 & 0.276202 & 0.466326 \\
\hline 50 & $1-13 \quad 14-15 \quad 16-30$ & 0.248869 & 0.25005 & 0.24218 & 105 & $1-45-1011-30$ & 0.107886 & 0.116189 & 0.060836 \\
\hline 51 & $1-34-89-30$ & 0.111145 & 0.119242 & 0.065259 & 106 & $1-910-15 \quad 16-30$ & 0.0808 & 0.068292 & 0.151676 \\
\hline 52 & $1-78-2425-30$ & 1.0087 & 0.615327 & 3.237814 & 107 & $1-8 \quad 9-12 \quad 13-30$ & 0.065657 & 0.056708 & 0.116364 \\
\hline 53 & $1-15 \quad 16-25 \quad 26-30$ & 0.286123 & 0.283774 & 0.299432 & 108 & $1-10$ 11-11 $12-30$ & 0.12038 & 0.111026 & 0.17339 \\
\hline 54 & $1-910-1011-30$ & 0.112258 & 0.108331 & 0.134512 & 109 & $1-78-2728-30$ & 0.253128 & 0.126684 & 0.969646 \\
\hline 55 & 1-15 $16-2930-30$ & 0.292235 & 0.283441 & 0.342071 & 110 & $1-13 \quad 14-14 \quad 15-30$ & 0.254633 & 0.255727 & 0.248431 \\
\hline
\end{tabular}

Table 2 shows the fitness values for 110 group structures along the PSO random walk path calculated by PyGroup, while the entire solution space contains 406 possible group structures. The calculated group structure fitness values are stored as a dictionary in PyGroup to avoid repeated simulations. The average of the overall fitness for the 110 entries in the dictionary is 0.218 , compared to the optimal fitness 0.0559 in Table 1.

To further reduce the computation cost, we also built a 2-D DRCC model as the basis for PyGroup group structure optimization. The total number of fine meshes in the 2-D model reduces to 5220, as shown in Figure. 2. 


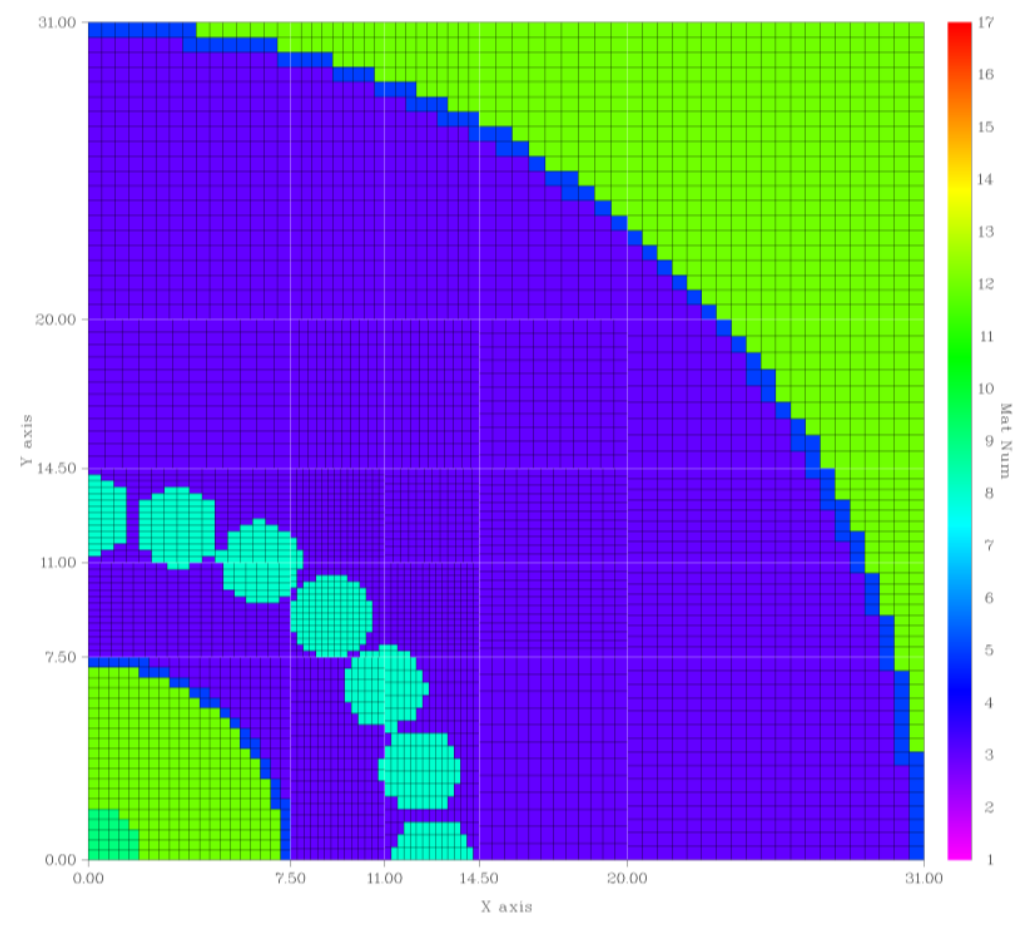

Figure 2. Meshing plot of the 2-D DRCC model.

PyGroup found the same optimal group structure for the 2-D model as shown in Table 3.

Table 3. Fitness metrics of the optimal 3-BG structure for the 2-D DRCC model.

\begin{tabular}{lllll}
\hline Optimal 3-BG structure: & \multicolumn{5}{c}{$1-78-12$} & $13-30$ \\
Overall fitness: & 0.047 & & \\
Response Rel. Err.(RMS fwd adj) : & 0.0369 & 0.0346 & 0.039 \\
Flux Rel. Err. (RMS fwd adj) : & 0.1047 & 0.1052 & 0.1043 \\
\hline Forward Flux Rel. Err: Material Name:(RMS, BG number) \\
Mat. Name & RMS & BG_1 & BG_2 & BG_3 \\
Polyethylene & $12.84 \%$ & $18.86 \%$ & $11.68 \%$ & $1.53 \%$ \\
Al & $9.77 \%$ & $14.34 \%$ & $8.19 \%$ & $3.67 \%$ \\
he3-4atm & $12.81 \%$ & $19.27 \%$ & $10.21 \%$ & $4.06 \%$ \\
Water & $10.08 \%$ & $6.99 \%$ & $1.83 \%$ & $15.90 \%$ \\
Air-humid & $7.62 \%$ & $11.09 \%$ & $6.28 \%$ & $3.40 \%$ \\
\hline Adjoint Flux Rel. Err: Material Name:(RMS, BG number) \\
Mat. Name & RMS & BG_1 & BG_2 & BG_3 \\
Polyethylene & $7.57 \%$ & $8.72 \%$ & $7.70 \%$ & $6.03 \%$ \\
Al & $10.39 \%$ & $9.36 \%$ & $9.30 \%$ & $12.24 \%$ \\
he3-4atm & $5.61 \%$ & $6.61 \%$ & $3.21 \%$ & $6.36 \%$ \\
Water & $13.92 \%$ & $2.50 \%$ & $13.64 \%$ & $19.72 \%$ \\
Air-humid & $9.33 \%$ & $7.13 \%$ & $8.64 \%$ & $11.65 \%$ \\
\hline
\end{tabular}

The overall fitness value for the 2-D model is also similar to the 3 -D model ( 0.047 versus 0.0559$)$. We note that the relative differences for fluxes and responses are similar in the 3-D model fitness metrics (0.0554 and 0.0587). In the 2-D model, detector response results agrees better (0.0369), while 
the fluxes shows larger difference (0.1047). The maxim errors are close to $20 \%$ for both forward and adjoint fluxes. However, the flux errors tend to compensate each other after integrating, as indicated by the response difference.

Table 4 3-BG structure fitness dictionary for 2-D DRCC model

\begin{tabular}{|c|c|c|c|c|c|c|c|c|c|}
\hline \# & BG structure & Fitness & Resp. RMS & Flux. RMS & $\#$ & BG structure & Fitness & Resp. RMS & Flux. RMS \\
\hline 1 & $1-78-89-30$ & 0.081462 & 0.084661 & 0.063334 & 52 & $\begin{array}{llll}1-5 & 6-9 & 10-30\end{array}$ & 0.090399 & 0.096874 & 0.053704 \\
\hline 2 & $1-3 \quad 4-9 \quad 10-30$ & 0.096909 & 0.100363 & 0.077333 & 53 & $1-2627-27 \quad 28-30$ & 0.755296 & 0.431627 & 2.589421 \\
\hline 3 & $1-12 \quad 13-2324-30$ & 0.203592 & 0.202113 & 0.21197 & 54 & $\begin{array}{llll}1-8 & 9-9 & 10-30\end{array}$ & 0.085139 & 0.081464 & 0.105964 \\
\hline 4 & $1-67-16 \quad 17-30$ & 0.086651 & 0.091374 & 0.059885 & 55 & $1-78-2021-30$ & 0.11113 & 0.103305 & 0.155469 \\
\hline 5 & $1-23-56-30$ & 0.211452 & 0.231822 & 0.096021 & 56 & $1-78-22 \quad 23-30$ & 0.135451 & 0.112043 & 0.268096 \\
\hline 6 & $1-2829-2930-30$ & 0.807905 & 0.432252 & 2.936605 & 57 & $1-56-67-30$ & 0.163226 & 0.177171 & 0.084206 \\
\hline 7 & $1-15 \quad 16-1920-30$ & 0.243636 & 0.250125 & 0.206866 & 58 & $1-18$ 19-19 20-30 & 0.330419 & 0.340459 & 0.27353 \\
\hline 8 & $1-56-14 \quad 15-30$ & 0.109661 & 0.116393 & 0.071511 & 59 & $1-34-13 \quad 14-30$ & 0.18018 & 0.188218 & 0.134633 \\
\hline 9 & $1-12-11 \quad 12-30$ & 0.153091 & 0.151603 & 0.161522 & 60 & $1-89-22 \quad 23-30$ & 0.115154 & 0.094031 & 0.234855 \\
\hline 10 & $1-67-15$ 16-30 & 0.080576 & 0.085093 & 0.054978 & 61 & $1-89-11 \quad 12-30$ & 0.055429 & 0.047031 & 0.103017 \\
\hline 11 & $1-11 \quad 12-12 \quad 13-30$ & 0.130094 & 0.116749 & 0.205712 & 62 & $1-2021-2122-30$ & 0.403947 & 0.398761 & 0.43334 \\
\hline 12 & $1-12-22 \quad 23-30$ & 0.449259 & 0.430184 & 0.557348 & 63 & $1-67-2930-30$ & 0.338672 & 0.174742 & 1.267611 \\
\hline 13 & $1-34-45-30$ & 0.234008 & 0.257572 & 0.100479 & 64 & $1-16 \quad 17-17 \quad 18-30$ & 0.266934 & 0.272299 & 0.236528 \\
\hline 14 & $1-67-78-30$ & 0.113082 & 0.120042 & 0.07364 & 65 & $1-10 \quad 11-12 \quad 13-30$ & 0.077553 & 0.061365 & 0.169285 \\
\hline 15 & $1-67-10 \quad 11-30$ & 0.075486 & 0.080607 & 0.04647 & 66 & $1-23-78-30$ & 0.10818 & 0.115651 & 0.065847 \\
\hline 16 & $1-45-56-30$ & 0.211309 & 0.23142 & 0.09735 & 67 & $1-910-2021-30$ & 0.103785 & 0.092766 & 0.166229 \\
\hline 17 & $1-11 \quad 12-16 \quad 17-30$ & 0.121898 & 0.108116 & 0.199997 & 68 & $\begin{array}{lll}1-1 & 2-18 & 19-30\end{array}$ & 0.322418 & 0.344726 & 0.196005 \\
\hline 18 & $1-34-11 \quad 12-30$ & 0.13146 & 0.132837 & 0.123658 & 69 & $1-8$ 9-18 19-30 & 0.073639 & 0.065681 & 0.118736 \\
\hline 19 & $1-78-17 \quad 18-30$ & 0.066663 & 0.06591 & 0.07093 & 70 & $1-45-12 \quad 13-30$ & 0.11229 & 0.118399 & 0.077668 \\
\hline 20 & $1-7 \quad 8-11 \quad 12-30$ & 0.049307 & 0.049213 & 0.049838 & 71 & $1-13 \quad 14-2526-30$ & 0.234613 & 0.229749 & 0.262173 \\
\hline 21 & $1-45-2930-30$ & 0.454547 & 0.257616 & 1.57049 & 72 & $1-13 \quad 14-17 \quad 18-30$ & 0.217544 & 0.21844 & 2468 \\
\hline 22 & $1-9 \quad 10-13 \quad 14-30$ & 0.056527 & 0.042465 & 0.136211 & 73 & $1-67-14 \quad 15-30$ & 0.077033 & 0.0814 & 0.052289 \\
\hline 23 & $1-34-78-30$ & 0.110718 & 0.119093 & 0.063256 & 74 & $1-23 \quad 24-24 \quad 25-30$ & 0.515079 & 0.430604 & 0.99377 \\
\hline 24 & $1-10$ 11-18 $19-30$ & 0.097529 & 0.084931 & 0.168914 & 75 & $1-23-34-30$ & 0.326519 & 0.357153 & 0.152928 \\
\hline 25 & $1-12 \quad 13-13 \quad 14-30$ & 0.19478 & 0.189664 & 0.223768 & 76 & $1-10 \quad 11-13 \quad 14-30$ & 0.075466 & 0.058798 & 0.169924 \\
\hline 26 & $1-12-23-30$ & 0.393215 & 0.42946 & 0.187827 & 77 & $1-8 \quad 9-15 \quad 16-30$ & 0.048667 & 0.038047 & 0.108845 \\
\hline 27 & $1-78-13 \quad 14-30$ & 0.046899 & 0.045708 & 0.053648 & 78 & $1-17 \quad 18-18 \quad 19-30$ & 0.299934 & 0.309937 & 0.24325 \\
\hline 28 & 1-1 2-9 $10-30$ & 0.101081 & 0.099708 & 0.108866 & 79 & 1-7 8-12 13-30 & 0.046115 & 0.045147 & 0.051602 \\
\hline 29 & $1-34-15 \quad 16-30$ & 0.196979 & 0.207276 & 0.138628 & 80 & $1-78-16 \quad 17-30$ & 0.053403 & 0.052289 & 0.059715 \\
\hline 30 & $1-11 \quad 12-1920-3$ & 0.138604 & & & 81 & $1-15 \quad 16-2021-30$ & & 0.253251 & 0.206605 \\
\hline 31 & $1-67-13 \quad 14-30$ & 0.07538 & 0.07964 & 0.051241 & 82 & $1-7 \quad 8-18 \quad 19-30$ & 0.080025 & 0.078705 & 0.087504 \\
\hline 32 & $1-9$ 10-16 17-30 & 0.060946 & 0.046987 & 0.140045 & 83 & $1-10 \quad 11-2021-30$ & 0.116257 & 0.104738 & 0.181533 \\
\hline 33 & $1-78-2122-30$ & 0.12676 & 0.110609 & 0.218281 & 84 & $1-14 \quad 15-17 \quad 18-30$ & 0.226778 & 0.228727 & 0.215735 \\
\hline 34 & $1-89-14 \quad 15-30$ & 0.047093 & 0.036485 & 0.107205 & 85 & $1-12 \quad 13-2122-30$ & 0.200301 & 0.200286 & 0.200388 \\
\hline 35 & $1-2122-22 \quad 23-30$ & 0.450955 & 0.420422 & 0.623971 & 86 & $1-15 \quad 16-16 \quad 17-30$ & 0.247945 & 0.248719 & 0.243563 \\
\hline 36 & $1-10$ 11-14 15-30 & 0.075582 & 0.058859 & 0.170345 & 87 & $1-23-10 \quad 11-30$ & 0.118987 & 0.115965 & 0.136114 \\
\hline 37 & $1-11 \quad 12-13 \quad 14-30$ & 0.123475 & 0.109522 & 0.20254 & 88 & $1-56-2425-30$ & 0.274007 & 0.225377 & 0.54958 \\
\hline 38 & $1-12-67-30$ & 0.160554 & 0.174752 & 0.080097 & 89 & $1-12 \quad 13-2021-30$ & 0.196672 & 0.196935 & 0.195179 \\
\hline 39 & $1-9$ 10-11 $12-30$ & 0.068003 & 0.056426 & 0.133606 & 90 & $1-1920-2021-30$ & 0.354944 & 0.363467 & 0.306646 \\
\hline 40 & $1-16$ 17-26 27-30 & 0.275737 & 0.277152 & 0.267722 & 91 & $1-11 \quad 12-14 \quad 15-30$ & 0.122237 & 0.108159 & 0.202015 \\
\hline 41 & $1-7 \quad 8-9$ 10-30 & 0.073007 & 0.075699 & 0.057753 & 92 & $1-12 \quad 13-17 \quad 18-30$ & 0.188414 & 0.185547 & 0.204663 \\
\hline 42 & $1-78-15$ 16-30 & 0.049662 & 0.048499 & 0.056255 & 93 & $1-10 \quad 11-22 \quad 23-30$ & 0.129593 & 0.113079 & 0.223173 \\
\hline 43 & $1-2728-2829-30$ & 0.773559 & 0.432513 & 2.706148 & 94 & $1-45-1011-30$ & 0.092005 & 0.097815 & 0.05908 \\
\hline 44 & $1-78-14 \quad 15-30$ & 0.047667 & 0.046475 & 0.054423 & 95 & $1-89-12 \quad 13-30$ & 0.047038 & 0.036854 & 0.104742 \\
\hline 45 & $1-14 \quad 15-15 \quad 16-30$ & 0.233216 & 0.232146 & 0.239277 & 96 & $\begin{array}{lll}1-8 & 9-13 & 14-30\end{array}$ & 0.046433 & 0.035814 & 0.106605 \\
\hline 46 & $1-67-2627-30$ & 0.306677 & 0.174662 & 1.054766 & 97 & $1-12 \quad 13-2526-30$ & 0.213885 & 0.203208 & 0.274384 \\
\hline 47 & $1-14 \quad 15-18 \quad 19-30$ & 0.226754 & 0.230535 & 0.205324 & 98 & $1-89-10 \quad 11-30$ & 0.072525 & 0.067274 & 0.102279 \\
\hline 48 & $1-2021-25 \quad 26-30$ & 0.419648 & 0.403671 & 0.510187 & 99 & $1-2 \quad 3-9 \quad 10-30$ & 0.100859 & 0.099521 & 0.108444 \\
\hline 49 & $1-12-45-30$ & 0.234645 & 0.25784 & 0.103205 & 100 & $1-78-1011-30$ & 0.063674 & 0.065663 & 0.0524 \\
\hline 50 & $1-9$ 10-10 11-30 & 0.088846 & 0.080853 & 0.134143 & 101 & $1-13 \quad 14-14 \quad 15-30$ & 0.227651 & 0.225628 & 0.239111 \\
\hline 51 & $1-56-2021-30$ & 0.210218 & 0.210026 & 0.211308 & 102 & $1-10 \quad 11-11 \quad 12-30$ & 0.093033 & 0.07925 & 0.171133 \\
\hline
\end{tabular}

Similar to the 3-D model group structure dictionary, PyGroup/SDFF also generated a 102-entry dictionary (Table 4) based on the 2-D model with an average fitness of 0.184 . For both dictionaries, 
each entry requires a pair of forward and adjoint transport simulations by the TITAN code. For the 3-D model, on average it takes about $\sim 700 \mathrm{sec}$ for each forward transport simulation on the PACE/Critcel cluster using 8 processors, while each adjoint run costs $\sim 1100 \mathrm{sec}$. By comparison, it usually takes less than one minute for the 2-D model.

\subsection{Comparison of YGROUP/PBFA with PyGroup/SDFF}

In this section, PyGroup/SDEF was used to provide the reference solutions to examine the performance of YGROUP/PBFA with the 2-D DRCC model. We evaluated the PBFA values for the group structures in the 3-BG dictionary generated by PyGroup/SDFF (Table 4). The results are compared in Figure 3.

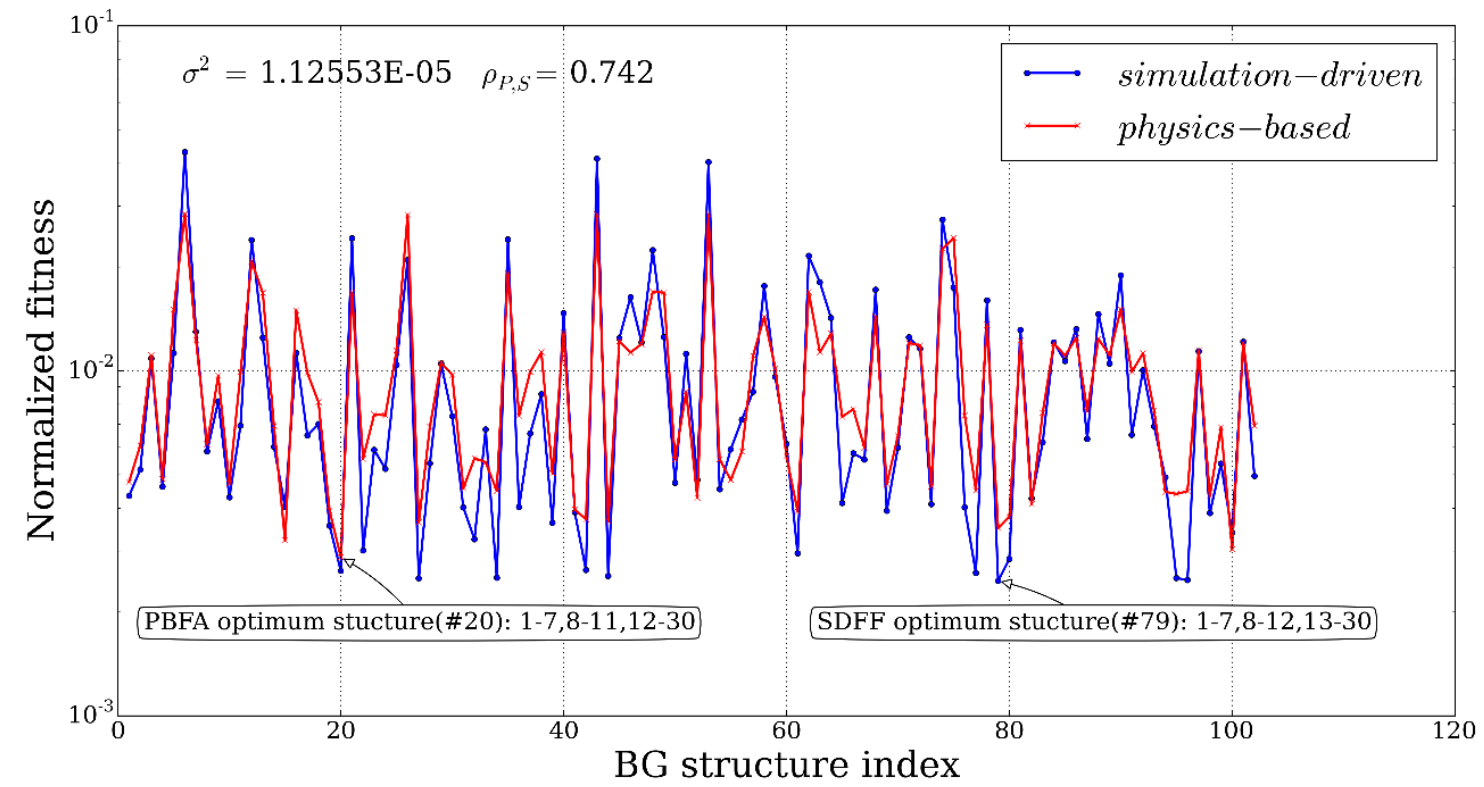

Figure 3. Normalized PBFA and SDFF fitness comparison for the 3-BG structure dictionary

In Figure 3, we normalized the PBFA and SDFF fitness values to unity for all the 102 3-BG structures in the dictionary, respectively. Figure 3 shows that their fitness values generally agree with each other. The PBFA identifies a slight different group structure, 1-7, 8-11, 12-30, as the global optimum, while the SDFF global optimal structure is: 1-7, 8-12, 13-30. Note that we used unequal weights in Eq. 1 for the detector response $\left(w_{0}=0.85\right)$ and flux $\left(w_{1}=0.15\right)$ terms in the SDFF evaluation. The weight parameters are set to bias toward the detector response, because the goal for this DRCC application is to conserve the detector response using fewer energy groups.

We also calculated the mean of squared difference $\sigma^{2}$, and the correlation coefficient $\rho_{P, S}$ of the PBFA and SDFF, defined as Eqs 44 and 45 respectively.

$$
\sigma^{2}=\frac{1}{N} \sum_{i=1}^{N}\left(P_{i}-S_{i}\right)^{2}
$$




$$
\rho_{P, S}=\frac{\operatorname{cov}(P, S)}{\sigma_{P} \sigma_{S}}=\frac{\sum_{i=1}^{N}\left(P_{i}-\frac{1}{N}\right)\left(S_{i}-\frac{1}{N}\right)}{\sqrt{\sum_{i=1}^{N}\left(P_{i}-\frac{1}{N}\right)^{2} \sum_{i=1}^{N}\left(S_{i}-\frac{1}{N}\right)^{2}}}
$$

where $P_{i}$ and $S_{i}$ are the $i$ 'th PBFA and SDFF evaluated fitness values, respectively; $N$ is the total number of group structures in the dictionary. $\sigma_{P}$ and $\sigma_{S}$ are the variances of the PBFA and SDFF, respectively; and $\operatorname{cov}(P, S)$ is the covariance of PBFA and SDFF. Note that the fitness function values are normalized independently. Therefore, their average values are: $E(P)=E(S)=\frac{1}{N}$. For the 3-BG dictionary shown in Figure 3, $\sigma_{3 B G}^{2}=1.12553 \times 10^{-5}$ and $\rho_{P, S}=0.74156$. While the $\sigma^{2}$ value is a measure of the average difference between PBFA and SDFF, the $\rho_{P, S}$ value depicts the strength of the correlation. Generally, the value of $\rho_{P, S}$ lies in between 0 and 1, with larger values represent higher correlation. Besides the 3-BG comparison, Figure 4 shows the PBFA and SDFF values for the 2, 4, 5, and 6 BGs dictionaries.

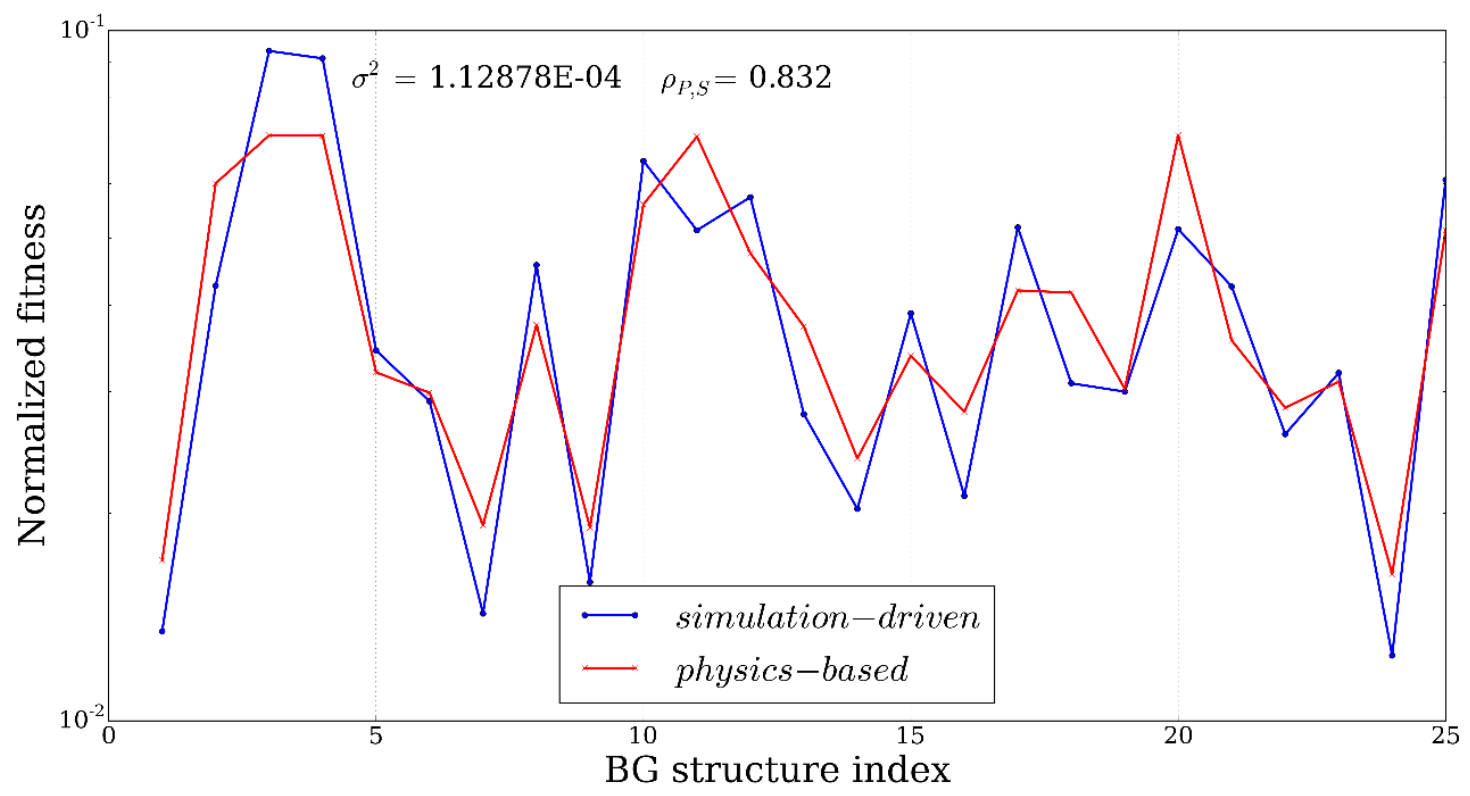

Figure 4a. Normalized PBFA and SDFF fitness value comparison for the 2-BG dictionary 


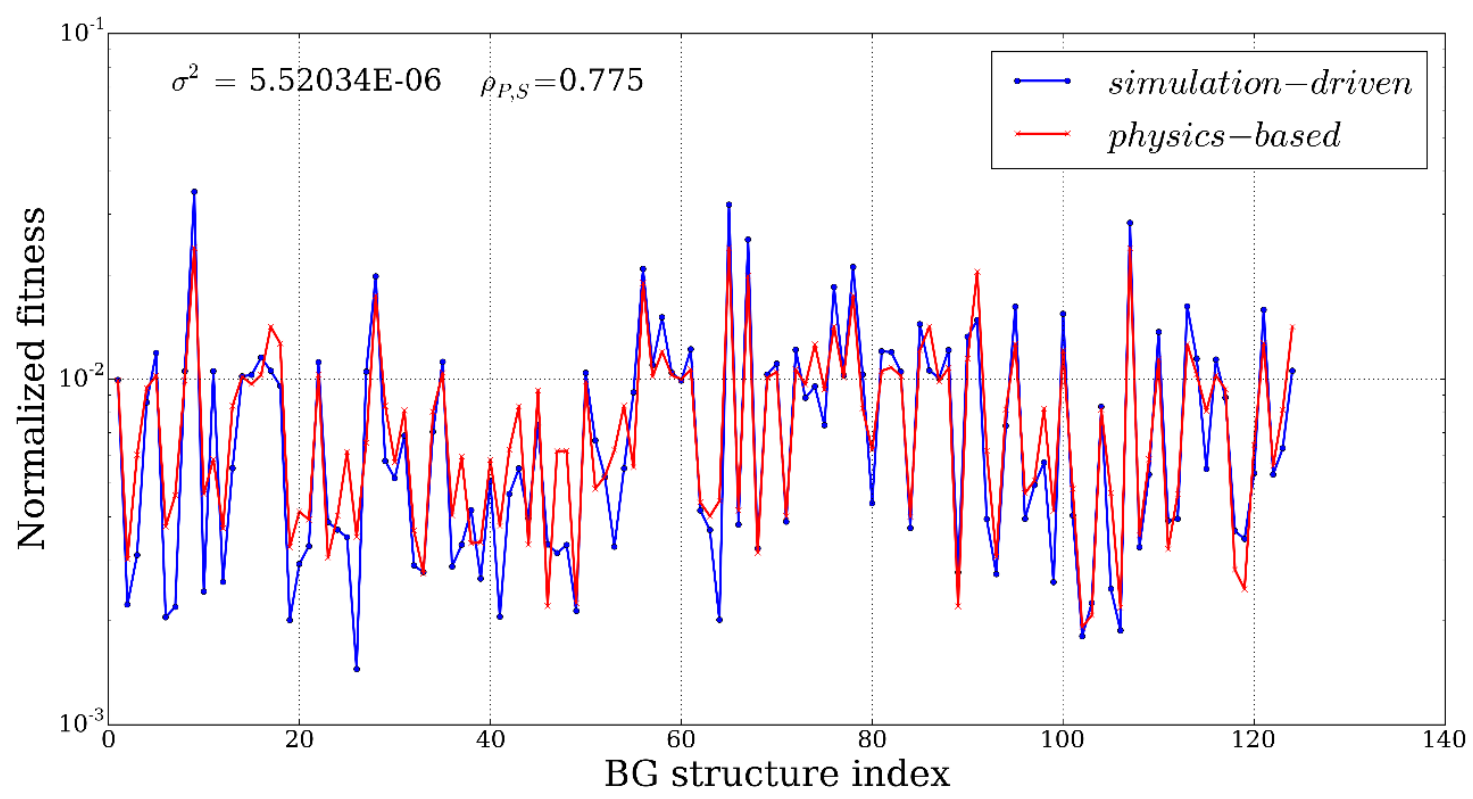

Figure 4b. Normalized PBFA and SDFF fitness value comparison for the 4-BG dictionary.

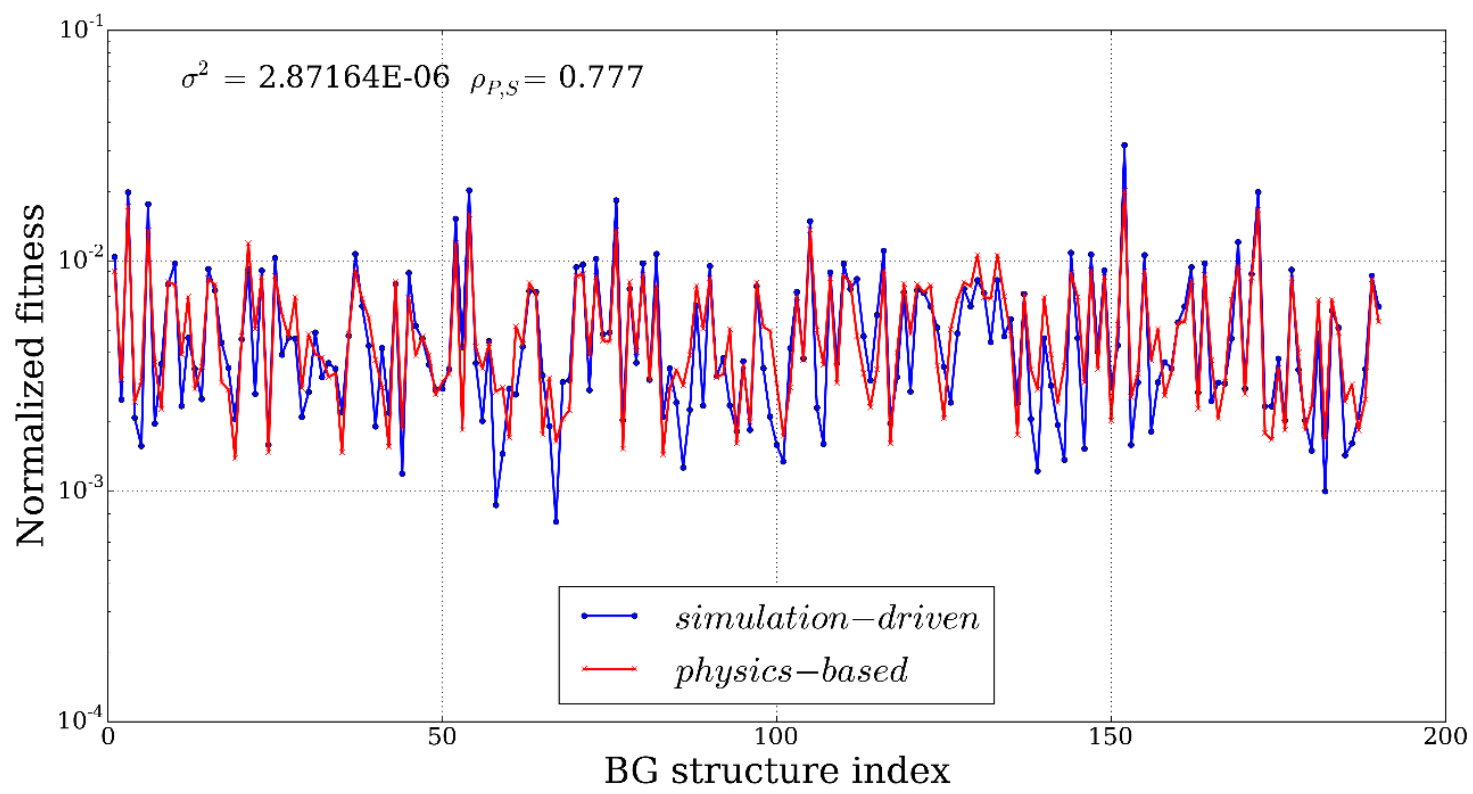

Figure 4c. Normalized PBFA and SDFF fitness value comparison for the 5-BG dictionary 


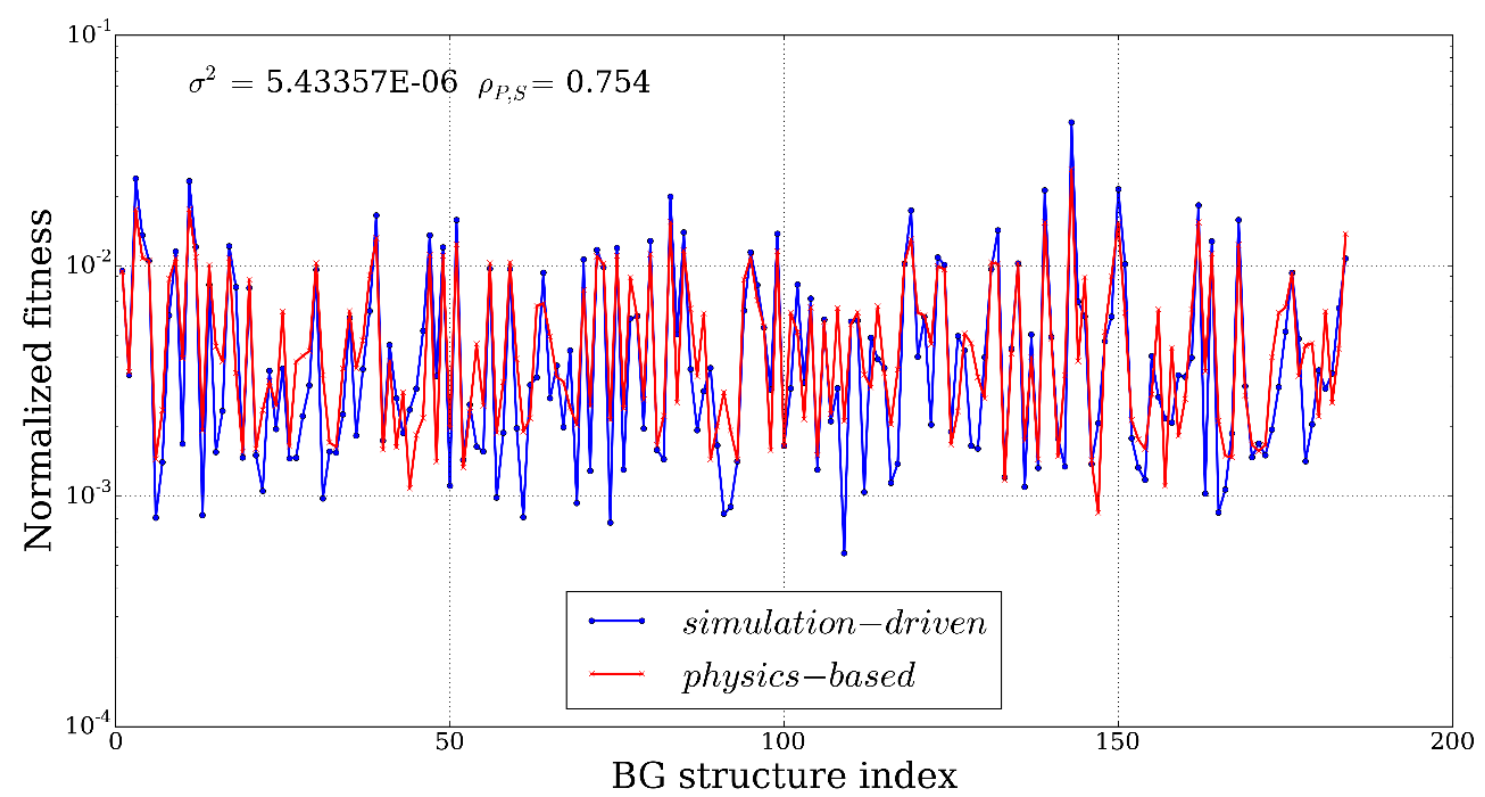

Figure 4d Normalized PBFA and SDFF fitness value comparison for the 6-BG dictionary

To further verify the correlation between PBFA and SDFF, we generated and examined more group structure dictionaries up to 15 BGs. The results are summarized in Table 5.

Table 5. Correlation of PBFA and SDFF for dictionaries from 2 to 15 BGs.

\begin{tabular}{cccc} 
\# BG & \# of entries & $\boldsymbol{\rho}_{\boldsymbol{P}, \boldsymbol{S}}$ & $\boldsymbol{\sigma}^{\mathbf{2}}$ \\
\hline 2 & 25 & 0.83232 & $1.12878 \mathrm{E}-04$ \\
3 & 102 & 0.74156 & $1.12553 \mathrm{E}-05$ \\
4 & 124 & 0.77542 & $5.52034 \mathrm{E}-06$ \\
5 & 190 & 0.77684 & $2.87164 \mathrm{E}-06$ \\
6 & 184 & 0.75386 & $5.43357 \mathrm{E}-06$ \\
7 & 62 & 0.78567 & $5.86797 \mathrm{E}-05$ \\
8 & 145 & 0.71398 & $1.22417 \mathrm{E}-05$ \\
9 & 267 & 0.78424 & $3.80127 \mathrm{E}-06$ \\
10 & 31 & 0.68718 & $2.69322 \mathrm{E}-04$ \\
11 & 8 & 0.70596 & $2.10279 \mathrm{E}-03$ \\
12 & 299 & 0.83183 & $5.70711 \mathrm{E}-06$ \\
13 & 362 & 0.79194 & $3.58177 \mathrm{E}-06$ \\
14 & 371 & 0.96960 & $6.47433 \mathrm{E}-06$ \\
15 & 212 & 0.90479 & $1.31707 \mathrm{E}-05$ \\
\hline
\end{tabular}

Table 5 shows a similar correlation for BG dictionaries ranging from 2 to 15 . The number of entries in the dictionaries are limited by the total number of possible group structures. As the number of BGs increases, the size of the solution space (number of possible group structures) also increases from 29 (2-BG) to 77,558,760 (15-BG). For example, the 2-BG dictionary covers 25 out of 29 possible 
group structures, while the 212-entry 15-BG dictionary only includes a small fraction of the solution space. We can adjust the size of a dictionary by changing the pre-defined tolerance in the PSO iteration scheme in PyGroup. To diversify dictionary size, larger tolerance limits are used in the 10-BG and 11BG cases, resulting in smaller size dictionaries. Their corresponding correlation coefficients are $\sim 0.70$. The 14-BG dictionary has the largest size (371 entries), as well as the largest correlation coefficient of $\sim 0.97$.

The computational time for calculating SDFF using the TITAN forward and adjoint simulations increases significantly as the number of BG increases. For example, a typical 2-D DRCC 2-BG adjoint simulation costs about $\sim 24 \mathrm{sec}$, while it takes $\sim 260 \mathrm{sec}$ for a 15-BG adjoint simulation on the PACE/Critcel cluster using 8 processors. For comparison, YGROUP/PBFA calculates PBFA values for all the dictionary entries in Table 5 (a total of 2382 group structures) within 1.0 second. This highlights the tremendous advantage of PBFA over SDFF in the aspect of computation cost.

\section{Summary and conclusions}

In this study, we developed a novel formulation, Physics-Based Fitness Approximation (PBFA), for heuristic optimization of the group structure when collapsing Fine-Group (FG) cross section libraries to Broad Group (BG) libraries. Both the Continuous-Energy (CE) and Multi-Group (MG) Contributon Transport Equation (CTE) can be derived by combining the corresponding forward and adjoint transport equations. In the process of deriving MG-CTE, we encountered two different definitions of the scattering cross section by applying the MG theory either on the forward and adjoint $\mathrm{CE}$ transport equations, or directly on the CE-CTE. The former results in the commonly seen fluxweighted group constants, where the reaction rate is conserved during the CE-to-MG and/or FG-to-BG collapsing process. The latter, interestingly, leads to a hybrid scheme weighted by the forward fluxes of the incident FGs, and the adjoint functions of the outgoing FGs, so that the neutron importance is conserved. We concluded that the difference in the scattering cross section formula is introduced by the MG approximation. Therefore, the magnitude of the difference can be used as a metric to evaluate the fitness of a given BG structure. Unlike the Simulation-Driven Fitness Function (SDFF) we presented in Section 2.1, no costly BG transport simulations are required for PBFA.

To verify our theory, we also conducted a detailed numerical test on the DRCC model using a 30FG library. The YGROUP/PBFA and PyGroup/SDFF codes are used to evaluate the fitness values of over 2000 BG group structures for libraries ranging from 2-BG to 15-BG. The results show that PBFA is strongly correlated to SDFF with an overall average correlation coefficient of 0.82 . YGROUP/PBFA calculates the fitness of a given group structure using FG simulation results (pre-calculated) and cross section data, almost instantly using a modern desktop computer. On the other hand, PyGroup/SDFF is subject to the number of BGs and the size of the problem model because of the requirement of BG transport simulations. Although a single BG simulation is generally much faster than the FG transport simulation, due to the large number of BG structures to be evaluated in PSO, it still could take hours, or days to complete all the fitness evaluation tasks for a large problem model even with the help of 
large-scale parallel computing. The difference in computational time highlights the significant advantage of a highly-correlated PBFA design over SDFF.

Our PBFA formulation provides a very creative method to evaluate a group structure. It also can be easily implemented and applied to other FG-to-BG collapsing applications. However, there is still room for improvements on strengthening the correlation between PBFA and SDFF. In the group structure dictionaries generated for the DRCC model, we found that the global optimal structure identified by YGROUP/PBFA does not always match the one selected by PyGroup/SDFF. Often PBFA and SDFF find similar, but different optimal group structures. It is possible to further improve their correlation by introducing a regularization term in the PFBF formulation (Eq. 43). One possible candidate is the inter-group neutron flow rate. Additionally, flow rates can be used to identify important group barriers. By keeping these FG barriers fixed in the PSO random walk process, the size of the solution space is effectively reduced, which will provide further improvement on the performance of the heuristic optimization.

\section{Acknowledgements}

This work was supported by National Nuclear Security Administration of Department of Energy (DE-NA0001737).

\section{References}

Akbari, M., Minuchehr, A., Zolfaghari, A., Khoshahval, F., 2012. An investigation for an optimized neutron energy-group structure in thermal lattices using Particle Swarm Optimization. Ann. Nucl. Energy 47, 53-61. doi:http://dx.doi.org/10.1016/j.anucene.2012.02.016

Askew, J.R., Fayers, F.J., Kemshell, P.B., 1966. A general description of the lattice code WIMS. J. Brit. Nucl. Energy Soc. 564.

Bell, S., Glasstone, G., 1970. Nuclear Reactor Theory. Van Nostrand Reinhold Co., New York.

Beni, G., Wang, J., 1993. Swarm intelligence in cellular robotic systems, in: Dario, P., Sandini, G., Aebischer, P. (Eds.), Robots and Biological Systems: Towards a New Bionics? Springer Berlin Heidelberg, Berlin, Heidelberg, pp. 703-712. doi:10.1007/978-3-642-58069-7_38

Duderstadt, J.J., Hamilton, L.J., 1976. Nuclear Reactor Analysis. Wiley.

Ensslin, N., Evans, M.L., Menlove, H.O., Swansen, J.E., 1978. Neutron coincidence counters for plutonium measurements. Nucl. Mater. Manag. 7, 43-65.

Jin, T., Oki, S., 2004. ORIGEN2 Cross-Section Libraries for Fast Reactors Based on JENDL-3.3.

Kennedy, J., Eberhart, R., 1995. Particle swarm optimization, in: Neural Networks, 1995. Proceedings., IEEE International Conference on. pp. 1942-1948 vol.4. doi:10.1109/ICNN.1995.488968

Kotz, S., Balakrishnan, N., Johnson, N.L., 2004. Continuous Multivariate Distributions, Models and Applications, Continuous Multivariate Distributions. Wiley.

Leszczynski, F., Aldama, D.L., A.Trkov, 2007. WIMS-D library update : final report of a coordinated research project. Vienna.

MacFarlane, R.E., Muir, D.W., Boicourt, R.M., Kahler, A.C., 2012. The NJOY Nuclear Data Processing Sysem, Version 2012.

Marleau, G., Roy, R., Hébert, A., 1994. DRAGON: A Collision Probability Transport Code for Cell and Supercell Calculations. Montréal, Québec.

ORNL, 2011. SCALE: A Comprehensive Modeling and Simulation Suite for Nuclear Safety Analysis and Design. Oak Ridge, TN. 
Painter, J.W., Gerstl, S.A.W., Pomraning, G.C., 1980. the theory of contributon transport. Los Alamos Scientific Lab., NM (USA).

Reilly, D., Ensslin, N., Smith Jr, H., Kreiner, S., 1991. Passive nondestructive assay of nuclear materials.

Varley, E.S., Mattingly, J., 2008. Rapid feynman-y synthesis: Kynea3 cross-section library development, in: Transactions of the American Nuclear Society. pp. 575-576.

White, J.E., Wright, R.Q., Ingersoll, D.T., Roussin, R.W., Greene, N.M., MacFarlane, R.E., 1994. Vitamin-B6: A fine-group cross section library based on ENDF/B-VI for radiation transport applications. American Nuclear Society, Inc., La Grange Park, IL (United States).

Yi, C., Haghighat, A., 2010. A Three-Dimensional Block-Oriented Hybrid Discrete Ordinates and Characteristics Method. Nucl. Sci. Eng. 164, 221-247.

Yi, C., Manalo, K., Huang, M., Chin, M., Edgar, C., Applegate, S., Sjoden, G., 2012. Optimization of a neutron detector design using adjoint transport simulation. American Nuclear Society - ANS; La Grange Park, IL (United States).

Yi, C., Sjoden, G., 2013. Energy group structure determination using particle swarm optimization. Ann. Nucl. Energy 56, 53-56.

Yi, C., Sjoden, G., Edgar, C., 2014. A Novel Hybrid Weighting Scheme for Multi-group Cross Section Collapsing, in: ANS PHYSOR.

Yi, C., Sjoden, G., Mattingly, J., Courau, T., 2010. Computationally optimized multi-group cross section data collapsing using the YGROUP code, in: International Conference on the Physics of Reactors 2010, PHYSOR 2010. pp. 527-542. 